\title{
Network Asynchrony Underlying Increased Broadband Gamma Power
}

\author{
${ }^{\circledR}$ Nicolas Guyon, ${ }^{1}{ }^{\circledR}$ Leonardo Rakauskas Zacharias, ${ }^{2}{ }^{\circledR}$ Eliezyer Fermino de Oliveira, ${ }^{3,4}{ }^{\circledR}$ Hoseok Kim, ${ }^{1}$ \\ ${ }^{\circledR}$ João Pereira Leite, ${ }^{2}{ }^{\circledR}$ Cleiton Lopes-Aguiar, ${ }^{5}$ and ${ }^{\circledR}$ Marie Carlén ${ }^{1,6}$ \\ ${ }^{1}$ Department of Neuroscience, Karolinska Institutet, Stockholm, 17177, Sweden, ${ }^{2}$ Department of Neuroscience and Behavioral Sciences, Ribeirão \\ Preto Medical School, Universidade de São Paulo, Ribeirão Preto, 14049-900, Brazil, ${ }^{3}$ Center for Mathematics, Computing and Cognition- \\ Universidade Federal do ABC, São Bernardo do Campo, 09606-070, Brazil, ${ }^{4}$ Dominick P. Purpura Department of Neuroscience, Albert Einstein \\ College of Medicine, Bronx, New York 10461, ${ }^{5}$ Department of Physiology and Biophysics, Institute of Biological Sciences, Universidade Federal de \\ Minas Gerais, Belo Horizonte, 31270-901, Brazil, and ${ }^{6}$ Department of Biosciences and Nutrition, Karolinska Institutet, Huddinge, 14183, Sweden
}

Synchronous activity of cortical inhibitory interneurons expressing parvalbumin (PV) underlies expression of cortical $\gamma$ rhythms. Paradoxically, deficient PV inhibition is associated with increased broadband $\gamma$ power in the local field potential. Increased baseline broadband $\gamma$ is also a prominent characteristic in schizophrenia and a hallmark of network alterations induced by NMDAR antagonists, such as ketamine. Whether enhanced broadband $\gamma$ is a true rhythm, and if so, whether rhythmic PV inhibition is involved or not, is debated. Asynchronous and increased firing activities are thought to contribute to broadband power increases spanning the $\gamma$ band. Using male and female mice lacking NMDAR activity specifically in PV neurons to model deficient PV inhibition, we here show that neuronal activity with decreased synchronicity is associated with increased prefrontal broadband $\gamma$ power. Specifically, reduced spike time precision and spectral leakage of spiking activity because of higher firing rates (spike "contamination") affect the broadband $\gamma$ band. Desynchronization was evident at multiple time scales, with reduced spike entrainment to the local field potential, reduced cross-frequency coupling, and fragmentation of brain states. Local application of $S(+)$-ketamine in (control) mice with intact NMDAR activity in PV neurons triggered network desynchronization and enhanced broadband $\gamma$ power. However, our investigations suggest that disparate mechanisms underlie increased broadband $\gamma$ power caused by genetic alteration of PV interneurons and ketamine-induced power increases in broadband $\gamma$. Our study confirms that enhanced broadband $\gamma$ power can arise from asynchronous activities and demonstrates that long-term deficiency of PV inhibition can be a contributor.

Key words: asynchrony; broadband gamma; DOWN and UP states; NMDAR; parvalbumin; PFC

Significance Statement

Brain oscillations are fundamental to the coordination of neuronal activity across neurons and structures. $\gamma$ oscillations (30$80 \mathrm{~Hz}$ ) have received particular attention through their association with perceptual and cognitive processes. Synchronous activity of inhibitory parvalbumin (PV) interneurons generates cortical $\gamma$ oscillation, but, paradoxically, PV neuron deficiency is associated with increases in $\gamma$ oscillations. We here reconcile this conundrum and show how deficient PV inhibition can lead to increased and asynchronous excitatory firing, contaminating the local field potential and manifesting as increased $\gamma$ power. Thus, increased $\gamma$ power does not always reflect a genuine rhythm. Further, we show that ketamine-induced $\gamma$ increases are caused by separate network mechanisms.

Received Aug. 22, 2020; revised Jan. 21, 2021; accepted Jan. 30, 2021.

Author contributions: N.G., J.P.L., C.L.-A., and M.C. designed research; N.G. and C.L.-A. performed research; N.G., L.R.Z., E.F.d.O., H.K., and C.L.-A. analyzed data; N.G., L.R.Z., E.F.d.O., C.L.-A., and M.C. wrote the first draft of the paper; N.G., L.R.Z., E.F.d.O., J.P.L., C.L.-A., and M.C. edited the paper; N.G., L.R.Z., C.L.-A., and M.C. wrote the paper.

This work was supported in part by STINT Program Joint Brazilian-Swedish Research Collaboration Grant BR2014-5869, CAPES-STINT Program Grant 99999.009883/2014-02, European Research Council Starting Grant 337069, and Coordenação de Aperfeiçoamento de Pessoal de Nível Superior-Brasil (CAPES)-Finance Code 001. C.L.-A. was supported by São Paulo Research Foundation FAPESP Grant 2012/07107-2. M.C. was supported by Wallenberg Academy Fellow in Medicine Grant KAW 2012.0131 from the Knut and Alice Wallenberg
Foundation, Swedish Research Council 2016-02700, and Karolinska Institutet 2016-00139. We thank Flávio A. G. Mourão and Geraldo Cássio dos Reis for help with data and statistical analysis; and Hyeyoung Shin, Christopher Moore, Rodrigo Pena, Stephanie Rogers, and Adriano Tort for the valuable comments on this manuscript.

The authors declare no competing financial interests.

Correspondence should be addressed to Cleiton Lopes-Aguiar at cleiton-aguiar@ufmg.br or Marie Carlén at marie.carlen@ki.se.

https://doi.org/10.1523/JNEUROSCI.2250-20.2021

Copyright $\odot 2021$ the authors 


\section{Introduction}

Perisomatic parvalbumin (PV) inhibition is central to the coordinated interaction of excitation and inhibition that underlies the emergence of $\gamma$ oscillations, synchronized rhythmic high-frequency $(30-80 \mathrm{~Hz})$ patterns of electrical activity observed in the local field potential (LFP) (Buzsáki and Wang, 2012). Cortical $\mathrm{PV}$ interneurons receive NMDAR-dependent excitatory input from pyramidal neurons, but paradoxically, mutant mice lacking NMDAR activity in PV-expressing neurons (PV-Cre/NR1f/f mice) and other mouse models of PV neuron dysfunctions (del Pino et al., 2013; Cho et al., 2015) display increased power of spontaneous $\gamma$ oscillations (Korotkova et al., 2010; Carlén et al., 2012; Billingslea et al., 2014). We hereafter refer to LFP activities spanning across the $30-80 \mathrm{~Hz}$ band as broadband $\gamma$ (oscillations). Administration of NMDAR antagonists, such as ketamine (Pinault, 2008; Hakami et al., 2009; Kulikova et al., 2012; Caixeta et al., 2013; McNally et al., 2020) or MK-801 (Carlén et al., 2012; Molina et al., 2014; Hudson et al., 2020), is also associated with increased power in spontaneous broadband $\gamma$ in both anesthetized and awake rodents, and also in humans (Rivolta et al., 2015). Importantly, lack of NMDAR in PV neurons blunts this response (Carlén et al., 2012; Picard et al., 2019; Hudson et al., 2020), identifying PV interneurons as a central target of NMDAR antagonists. However, it is unclear whether pharmacologically induced enhancement of broadband $\gamma$ power is distinct from the increased spontaneous broadband $\gamma$ caused by PV neuron dysfunction.

Computational modeling has suggested that decreased excitability of PV interneurons because of an absence of slow excitatory NMDA currents would lead to reduced sensitivity to excitatory inputs and the requirement of a more synchronous excitatory input for PV firing (Carlén et al., 2012). This "suppression boundary" mechanism (Börgers and Kopell, 2005) is modeled to enhance $\gamma$ oscillations over a broad frequency range (Carlén et al., 2012; Jadi et al., 2016). Independently, optogenetic experiments have demonstrated that enhanced excitability of excitatory neurons in the MPFC increases spontaneous broadband $\gamma$ power (Yizhar et al., 2011), suggesting that increased spontaneous broadband $\gamma$ power can reflect an overall increased activity in cortical circuits (Sohal and Rubenstein, 2019). In line with this, systemic administration of MK-801 has demonstrated an association between increased broadband $\gamma$ (here $30-90 \mathrm{~Hz}$ ) power and increased firing in the $\mathrm{MPFC}$ of rats (Molina et al., 2014). Notably, the spike trains become disorganized, and the synchronization of action potential firing decreases. This supports the view that increased $\gamma$ power might not (always) reflect a rhythm (a genuine oscillation) but could be a result of increases in uncorrelated synaptic potentials and neuronal discharges (Uhlhaas and Singer, 2015; see Buzsáki and Wang, 2012, and references therein); that is, spontaneous/baseline broadband $\gamma$ oscillations could be fundamentally different from $\gamma$ synchrony caused by rhythmic neuronal activity coordinated by PV inhibition (Sohal and Rubenstein, 2019). Of relevance, both modeling and experimental findings show that dysfunction of PV inhibition results in increased variability of cortical excitatory activity (Carlén et al., 2012; Jadi et al., 2016). The mechanism(s) by which asynchronous neuronal activity caused by deficient PV inhibition could manifest as increased broadband $\gamma$ activity has not been established.

Depending on the ongoing cortical state, $\gamma$ oscillations can be persistent (e.g., during wakefulness and rapid-eye-movement [REM] sleep) or nested to slower $(<4 \mathrm{~Hz})$ oscillations (e.g., during non-REM [NREM] sleep) (Steriade, 2006; McKenna et al.,
2017). This pattern of $\gamma$ modulation is also observed during the cyclic alternation between activated and deactivated states under urethane anesthesia (Clement et al., 2008). Systemic administration of ketamine alters the cyclic alternations and decreases the coupling between $\delta$ and $\gamma$ oscillations (Lopes-Aguiar et al., 2020). While PV inhibition is suggested to regulate state transitions (Zucca et al., 2017), it is largely unknown how deficiency in PV inhibition affects cortical states.

To directly investigate a connection between deficient PV inhibition, increased broadband $\gamma$ power, and asynchronous neuronal activity, we recorded LFP and single-unit activity in the mPFC of urethane-anesthetized mice lacking NMDAR activity in PV neurons (PV-Cre/NR1f/f mice). Our experiments revealed altered $\mathrm{mPFC}$ state transitions and asynchronies at multiple time scales in PV-Cre/NR1f/f mice during the deactivated state (a cortical state normally marked by synchronous activity), including reduced coupling of $\gamma$ oscillations to slower oscillations and increased temporal variability in the firing of excitatory neurons. Moreover, PV-Cre/NR1f/f mice displayed state events with increased and asynchronous neuronal activity, causing spectral leakage of spiking activity to contaminate, and increase the power in, the broadband $\gamma$ band and the high-frequency band $(\mathrm{HFB} ; 100-150 \mathrm{~Hz})$. While application of $\mathrm{S}(+)$-ketamine triggered a state with increased broadband $\gamma$ power, desynchronized LFP oscillations, altered spike-LFP entrainment, and increased firing in (control) mice with intact NMDAR activity in PV neurons, our analyses suggest that increased broadband $\gamma$ power induced by ketamine and increased broadband $\gamma$ power, resulting from deficient PV inhibition, are caused by different mechanisms.

\section{Materials and Methods}

Animals. All procedures were performed in accordance and in compliance with the guidelines of the Stockholm Municipal Committee for animal experiments and the Karolinska Institutet in Sweden (approval 43/14). To generate mice lacking NMDAR specifically in PV interneurons (PV-Cre-NR1f/f mice), PV-Cre knock-in mice (JAX stock \#008069, RRID:IMSR_JAX:008069) were crossed to mice carrying the "floxed" NR1 alleles (JAX stock \#005246, RRID:IMSR_JAX:005246), as previously characterized (Carlén et al., 2012). Anesthetized electrophysiology recordings were performed on adult (15-20 weeks of age) PV-Cre/NR1f/ $\mathrm{f}(n=3$ females, $n=1$ male) and PV-Cre mice $(n=6$ females, $n=2$ males). PV-Cre/NR1f/f and PV-Cre mice were bred at the Karolinska Institutet. Animals were housed in groups (3-5 mice/cage) using individually ventilated caging systems (Sealsafe plus, GM 500, Tecniplast), under standardized conditions with a $12 \mathrm{~h}$ light-dark cycle (light 7:00 A. M.), stable temperature $\left(20 \pm 1^{\circ} \mathrm{C}\right)$, and humidity (40\%-50\%) with access to food (R70 Standard Diet, Lactamin; $4.5 \%$ fat, $14.5 \%$ protein, $60 \%$ carbohydrates) and water ad libitum.

Viral injection. The injections were performed in a biosafety cabinet Class 2 with a sterile surgical environment. A micropipette (10 $\mu$ l graduated borosilicate glass capillary; Wiretrol I Calibrated Micropipettes, Drummond) prefilled with mineral oil was fixed into the Quintessential Stereotaxic Injector (Stoelting) and filled with $0.5 \mu \mathrm{l}$ of virus (titer $10^{12}$ ) dyed with Fast Green 2.5\% (Electron Microscopy Sciences). The mouse ( $\sim 8$ weeks old) was injected subcutaneously with the analgesic buprenorphine $(0.1 \mathrm{mg} / \mathrm{kg}$; Temgesic, Indivior Europe) $30 \mathrm{~min}$ before surgery. The mouse was placed on a heating blanket $\left(37^{\circ} \mathrm{C}\right)$ in the stereotaxic apparatus (Harvard Apparatus) and anesthetized with 2\% isoflurane in oxygen, and the eyes were covered with eye lubricant (Viscotears, Novartis). The mouse's reflexes were tested, and the isoflurane level was reduced to $1 \%$ or $1.5 \%$ throughout the surgery. The scalp was shaved, and lidocaine $(4 \mathrm{mg} / \mathrm{kg})$ was injected subcutaneously before skin incision. The connective tissue around the skull was gently removed as needed for clear viewing of the bregma. The craniotomy was performed 
unilaterally above the mPFC: AP: $1.8 \mathrm{~mm}$ anterior to bregma, ML: 0.3 $\mathrm{mm}$ lateral to midline. Sterile saline $0.9 \%$ was regularly applied over the skull while drilling to remove bone dust and control heat generation. The total diameter of the burr hole was rarely larger than the diameter of the drill bit $(\sim 100 \mu \mathrm{m})$. The dura was carefully opened using a syringe needle, and the micropipette tip was lowered until reaching the target location (prelimbic area (PL) of the mPFC; AP: $1.8 \mathrm{~mm}$; ML: $0.3 \mathrm{~mm}$; DV: $-1.4 \mathrm{~mm}$, relative to the surface); $0.5 \mu \mathrm{l}$ of AAV-DIO-ChR2mCherry (rAAV2/Ef1A-DIO-hChR2(H134R)-mCherry; $4 \times 10 \mathrm{e} 12$ viral particles/ml, from UNC Vector Core) was injected at a rate of $0.1 \mu \mathrm{l} /$ min. Mice used for control of optogenetic artifacts (PV-Cre; $n=4$ ) were injected with AAV-DIO-eYFP (rAAV5/EF1a-DIO-eYFP; $4 \times 10 \mathrm{e} 12$ particles/ml; UNC Vector Core). The micropipette was retained at the injection site for $10 \mathrm{~min}$ after injection and before retraction. The edges of the skin were pulled together and sealed with small amounts of cyanoacrylate glue (Vetbond Tissue Adhesive, Henry Schein Medical), and carprofen $(5 \mathrm{mg} / \mathrm{kg}$; Norocarp, Norbrook Laboratories) was given subcutaneously. The animal was monitored until completely recovered from the anesthesia. An additional dose of carprofen was administered $24 \mathrm{~h}$ after surgery.

Electrophysiological recordings, optogenetic stimulation, and ketamine injection. Five to 7 weeks after the virus injection, the mice were anesthetized with urethane $(1.1 \mathrm{~g} / \mathrm{kg}$ in sterile saline $0.9 \%$, i.p.) and fixed in a stereotaxic apparatus (Digital Stereotaxic with Dual Manipulators; Leica Microsystems) on a heating blanket (Harvard Apparatus) to maintain the body temperature at $37 \pm 0.5^{\circ} \mathrm{C}$ and kept under $0.25 \%$ isoflurane anesthesia for the course of the surgery. Using the above-described coordinates, we made a new craniotomy over the initial burr hole, large enough to allow positioning of an optical fiber (details below) and a silicon probe (A4x2-tet-5 mm-150-200-312-A32 Neuronexus) coupled to a 32-channel headstage preamplifier (Neuralynx) linked to a Digital Lynx 4SX acquisition systems (Neuralynx). Small amounts of saline $0.9 \%$ were continuously dropped on the craniotomy to prevent brain surface dryness. An additional craniotomy was made over the parietal cortex to fix a ground screw, used as a reference. Then, we carefully positioned the silicon probe in the target position (AP: $1.8 \mathrm{~mm}$ anterior to bregma, ML: $0.3 \mathrm{~mm}$ relative to midline, $\mathrm{DV}:-2 \mathrm{~mm})$. We monitored in real time the electrophysiological signal for $30 \mathrm{~min}$ to allow the brain tissue to recover and optimize the recording condition. Two different recordings were performed. First, $50 \mathrm{~min}$ of recording was performed to monitor different brain states induced by urethane. One PV-Cre/NR1f/f mouse was not included in this part of the analysis because of poor recording quality. Second, a $60 \mathrm{~min}$ recording was performed to assess prefrontal oscillatory and neuronal activity alterations induced by ketamine. In this new recording session, after moving the silicon probe (DV: $\sim 0.2 \mathrm{~mm}$ ), single-unit and LFP oscillations were monitored for $30 \mathrm{~min}$ before and after $\mathrm{S}(+)$-ketamine $(100 \mu \mathrm{g}$ in sodium chloride $0.9 \%$ solution) applied over the craniotomy where the silicon probe was implanted. One PV-Cre mouse was lost during this phase of the recording. The recordings were acquired using the Cheetah 5.0 acquisition and experiment control software (Neuralynx). Electrical signals were divided, preamplified $(1000 \times)$, and filtered for LFPs and single-unit activity. The biological signal was sampled at $32 \mathrm{kHz}$ and bandpass filtered between 600 and $6000 \mathrm{~Hz}$ to record spikes and between 0.5 and $500 \mathrm{~Hz}$ to record LFPs.

For optogenetic manipulations, we positioned an unjacketed optical fiber $(200 \mu \mathrm{m}$ core diameter; numerical aperture: 0.22; ThorLabs) 1.5 $\mathrm{mm}$ above the recording site using a tilted insertion (10-15 degrees of slope; $0.3 \mathrm{~mm}$ below the brain surface), to attenuate photoelectric artifacts by reducing the contact exposure to the light (Cardin et al., 2010). Optical stimulation was generated by a $473 \mathrm{~nm}$ laser source $(100 \mathrm{~mW}$; CNI Lasers) triggered through a data acquisition board (BNC-2110, National Instruments) controlled by a custom-made LabView (National Instruments) program. A broad range of light-stimulation frequencies $(8,16,24,32,40,48,60,80$, and $90 \mathrm{~Hz})$ was applied randomly in bouts of $3 \mathrm{~s}$ of $1 \mathrm{~ms}$ pulses width with $5 \mathrm{~mW}$. Laser power was measured at the optical fiber tip before each experiment by a PM100D power meter console (ThorLabs).

Tissue processing, immunostaining, and imaging. The mice were deeply anesthetized with pentobarbital $(60 \mathrm{mg} / \mathrm{ml}$; Apotek Produktion \&
Laboratorier) and transcardially perfused with $1 \times$ PBS (0.1 $\mathrm{M}, \mathrm{pH} 7.4)$ followed by $4 \%$ PFA in PBS $(0.1 \mathrm{M}, \mathrm{pH} 7.4)$. The perfused brain was removed from the skull and postfixed with $4 \%$ PFA in $0.1 \mathrm{M}$ PBS at $4^{\circ} \mathrm{C}$ for $16 \mathrm{~h}$. The brain was thoroughly washed in $0.1 \mathrm{~m}$ PBS and thereafter sectioned (40 $\mu \mathrm{m}$ thickness) using a vibratome (Leica VT1000, Leica Microsystems). The sections were stored in $1 \times \mathrm{PBS}$ at $4^{\circ} \mathrm{C}$.

Vibratome cut (free-floating) tissues were permeabilized for $1 \mathrm{~h}$ with $1 \times$ TBST $(0.3 \%$ Triton-X in $1 \times$ TBS), blocked for $1 \mathrm{~h}$ at room temperature with $10 \%$ normal donkey serum in $1 \times$ TBST, and thereafter incubated with primary antibodies (rabbit polyclonal anti-PV; catalog \#PV25, RRID:AB_10000344, Swant; at a dilution of 1:1000) in $1 \times$ TBST at room temperature for 12-24 h. The sections were thereafter washed 3 times in $1 \times$ TBST, and incubated for 3-5 h with a secondary antibody (AlexaFluor-488 anti-rabbit; catalog \#711-545-152, RRID:AB_2313584, Jackson ImmunoResearch Laboratories) at a dilution of 1:500 in 1× TBST. The sections were thereafter consecutively washed with $1 \times$ TBST, $1 \times$ TBS, and $1 \times$ PBS (10 min each). All sections were mounted on glass slides (Superfrost Plus, Thermo Fisher Scientific) and coverslipped using 50:50 Glycerol:1× PBS mixed with DAPI (added for visualization of nuclei).

Images were acquired at $10 \times$ or $20 \times$ magnification with a fluorescent microscope (Leica Microsystems DM6000B) connected to an OrcaFLASH 4.0 C11440 digital camera (Hamamatsu). Images were spatially registered to the Allen Mouse Brain Common Coordinate Framework version 3 (ABA_v3) reference atlas (Lein et al., 2007) using the QuickNII tool (RRID:SCR_016854) (Puchades et al., 2019) and SBA Composer (Bakker et al., 2015).

Data analysis. All data analysis was conducted using custom scripts written in MATLAB (The MathWorks).

Brain state classification. LFP oscillations were divided in $30 \mathrm{~s}$ epochs. Using a Gaussian mixture model (GMM), epochs of the LFP were classified in deactivated, activated, and transition (unclassified) states based on the power of the $0.5-2 \mathrm{~Hz}$ and $3-45 \mathrm{~Hz}$ frequency bands for an initial clustering. The classified epochs were manually refined by eliminating epochs at the cluster edge. The spectrum content of each state was analyzed to confirm proper classification. Transition epochs were discarded from the analysis.

UP state and DOWN state classification. DOWN and UP states during deactivated states were detected based on the $z$-scored average firing rate for each mouse, with individually tuned thresholds. Firing rates were calculated with a $0.01 \mathrm{~s}$ bin and a Gaussian window of $0.4 \mathrm{~s}$. Units with an average firing rate $<0.01 \mathrm{spike} / \mathrm{s}$ were not included. Analyses were conducted only with paired events, where paired events correspond to the most recent DOWN state around an UP state event. UP state events with no surrounding DOWN state were excluded.

Spectral analysis. Power spectral density (PSD) was estimated using Welch's method (MATLAB algorithm), with $1 \mathrm{~s}$ Hamming window, with $50 \%$ overlap and a 5120 FFT points. Normalized power was obtained by dividing the PSD estimation by the integrated power over all frequencies $(0.5-200 \mathrm{~Hz})$. For visualization of LFP oscillations in specific frequency bands, a bandpass filter using two-way least-squares FIR filtering (eegfilt function from EEGLAB toolbox) was applied (Delorme and Makeig, 2004).

Morlet wavelet spectrograms of the LFP oscillations were calculated using a wavelet convolution, built with a Morlet wavelet with a frequency resolution of $0.2 \mathrm{~Hz}$ and frequency bounds of 0.5 and $150 \mathrm{~Hz}$. Each frequency of the resultant spectrum was normalized by the sum of power spectrum from 0.5 to $200 \mathrm{~Hz}$. The power distribution over the frequency bands was calculated by the extraction of the amplitude envelope (Hilbert transform) from the wavelet-decomposed signal to obtain representative Morlet wavelets of different frequency bands (1-4, 6-12, 12$15,15-30,30-80,100-150 \mathrm{~Hz})$. The transition-triggered average field potential was calculated as the mean LFP during $4 \mathrm{~s}$ for all events, centered at the DOWN-to-UP state transition.

Phase-amplitude modulation index (MI). Phase-amplitude coupling was estimated by the MI as described by Tort et al. (2010). MI is a normalized measure that reflects how well the amplitude of a faster oscillation is phase-locked to an underlying lower frequency cycle. Comodulation maps were constructed calculating the MI at 
phase frequencies from 0.5 to $6 \mathrm{~Hz}$ (in steps of $1 \mathrm{~Hz}$ ) and amplitude frequencies from 30 to $150 \mathrm{~Hz}$ (in steps of $5 \mathrm{~Hz}$ ). Shannon entropy of the distribution of mean amplitudes per phase (divided into 18 bins) in each frequency bin was calculated to obtain the cross-frequency MI for each $30 \mathrm{~s}$ epoch. Specifically, the MI between the 0.5$2 \mathrm{~Hz}$ band and broadband $\gamma$, and between the $0.5-2 \mathrm{~Hz}$ band and $\mathrm{HFB}$, were calculated and then averaged for group comparisons.

Spectral entropy and high-frequency (HF) index. To quantify the frequency dispersion level in the PSD, we performed an analysis of the spectral entropy of the LFP $(30-150 \mathrm{~Hz})$. Based on previous work using this measure (Valero et al., 2017), we determined the spectral entropy (in bits) of the LFP by calculating the Shannon Entropy, given by Equation 1 as follows:

$$
H=-\log (\Sigma P(f) \log (P(f)))
$$

Where $P(f)$ is the normalized power spectrum calculated in 35 bins of frequency from 30 to $150 \mathrm{~Hz}$ band. The higher the spectral entropy value, the more uniformly the power is distributed across the PSD; that is, the power spectrum of the network firing rate is flat, as in the case of broadband noise activity. In contrast, the lower the spectral entropy value, the more concentrated the power is at one frequency (around a peak), as in the case of single-frequency network oscillations.

To quantify the level of spectral leakage by spiking activity contributing to the power of the LFP, we determined the HF index (with values from 0 to 1). We first calculated the power at $30-150 \mathrm{~Hz}$ and $200-300 \mathrm{~Hz}$ frequency bands by extracting the amplitude envelope (Hilbert transform) from the wavelet-decomposed signal and then divided the power at $200-300 \mathrm{~Hz}$ frequency band by the power at $30-150 \mathrm{~Hz}$. The closer to 1 the HF index value, the higher the level of spectral leakage by spiking activity contributes to the power of the LFP.

LFP autocorrelogram. To exemplify how ketamine changes the level of synchronicity of prefrontal oscillations, LFP autocorrelograms were calculated for all $30 \mathrm{~s}$ epochs. For this, we measured the similarity for each $30 \mathrm{~s}$ LFP and their respective shifted lags (from -2 to $2 \mathrm{~s}$ ), using the function $x$ corr from MATLAB with coeff normalization, thus obtaining cross-correlation arrays for each epoch. Then, we built a two-dimensional plot with the cross-correlation values for all epochs along time (60 min), indicating the autocorrelation values as a colormap.

Spike sorting. Spikes were manually sorted into individual units (putative neurons) offline based on analysis of peak amplitude, valley amplitude, peak-valley ratio, spike width, waveform energy, and principal components using the MClust 4.4 offline spike sorting software (A.D. Redish; available at http://redishlab.neuroscience. umn.edu/MClust/MClust.html). Cluster quality was evaluated by the isolation distance and L-ratio (Schmitzer-Torbert et al., 2005). Clusters with isolation distance $<12$ or L-ratio $>0.3$ were excluded. Autocorrelation and interspike interval (ISI) histogram charts were inspected for all putative neurons. In cases where the autocorrelation showed absolute refractory period violations (spike counts at periods $<1.2 \mathrm{~ms}$ ), we improved cluster separation; otherwise, the cluster was excluded.

Unit classification. The units were classified into wide-spiking (WS) putative pyramidal neurons and narrow-spiking (NS) putative interneurons based on the distribution of (1) the peak-to-valley ratio (the ratio between the amplitude of the initial peak and the following trough) and (2) the half-valley width of each spike waveform. For the objective classification of units between WS and NS, a GMM was fit to the unit's features, as previously described (H. Kim et al., 2016). Units with low classification confidence $(p>0.05)$ were discarded. To identify putative $\mathrm{PV}$ interneurons, the NS population was further classified based on the combination of waveform similarity index and the magnitude of the repolarization (spike waveform peak). The waveform similarity index $(r)$ was calculated by correlating each spike waveform with the mean spike waveform of light-activated units. The value of 1 indicates identical spike waveforms. NS neurons with a peak amplitude (normalized) $<0.8$ and high waveform similarity index $(>95 \%)$ were classified as PV interneurons.
Single-unit activity analyses. To determine single-unit activity during DOWN-to-UP state transitions, peri-event time histograms (PETH, $10 \mathrm{~ms}$ bins, $500 \mathrm{~ms}$ pre-event, $500 \mathrm{~ms}$ post-event) were calculated and convoluted with a Gaussian kernel of $50 \mathrm{~ms}$ and normalized using $z$ score. For each unit, we calculated the mean firing rate at DOWN and UP states, the latency to the peak (maximum firing rate; relative to the DOWN-to-UP transition), and the Full Width at Half Maximum (FWHM), which reflects the level of spike timing precision around the peak. Units with a firing rate $<0.1 \mathrm{spike} / \mathrm{s}$ were excluded from these analyses. To determine single-unit activity under ketamine, we calculated for each $30 \mathrm{~s}$ epoch ISI histograms for every unit to obtain the mean firing rate ( $\mathrm{FR}=1 / \mathrm{ISI})$ and the coefficient of variation $(\mathrm{CV})$, as the ratio of the Standard Deviation (SD) of ISI by the average ISI (CV $=\mathrm{SD}$ (ISI)/mean(ISI)). To assess the effects of optogenetic stimulation on the activation of mPFC neurons, neuronal activity relative to the light events was expressed in a PETH and a spike raster (rastergram) for each neuron. To dismiss any light artifacts in light-responsive units, the spike waveform shape similarity was determined by calculating the correlation coefficient $(r)$ between two average spike waveforms ( $1 \mathrm{~s}$ before and after the light onset) for each unit.

Measurement of spike-LFP entrainment using von Mises statistics. We quantified the entrainment of spikes to the LFP as previously described (Schomburg et al., 2014). In brief, we extracted the entrainment of spikes to the LFP by filtering the LFP with a second-order Butterworth filter and extracting the phase using the Hilbert transform. By calculating the phases of the filtered-LFP where spikes occurred, we tested whether the spike was significantly modulated by the LFP using a von Mises distribution (or circular normal distribution) statistics. The entrainment of spikes by the LFP was calculated on a log scale from 21 to $242 \mathrm{~Hz}$, and the mean length of the resultant vector was taken for each frequency and plotted in color code. To avoid spurious spike entrainment to high frequencies because of LFP contamination by the spike itself, we used the LFP on the shank next to the shank where the specific cell was recorded $(200 \mu \mathrm{m}$ distance). Units recorded from electrodes with noisy LFP signals were excluded. Units included in the analysis: WS units (PV-Cre: $n=22$; PV-Cre/NR1f/f: $n=90$ ), NS units (PVCre: $n=7$; PV-Cre/NR1f/f: $n=12$ ), and putative PV units (PV-Cre: $n=3$; PV-Cre/NR1f/f: $n=5$ ).

A bootstrap procedure was used for identification of significant differences in the spike-LFP entrainment between PV-Cre and PV-Cre$\mathrm{NR} 1 \mathrm{f} / \mathrm{f}$ mice (during baseline and in response to ketamine application, respectively). The spike-LFP entrainment for the two mouse cohorts was calculated in randomly shuffled LFP frequencies, and the proportion of neurons modulated for each frequency was recalculated. This procedure was repeated for 10,000 times and a Confidence Interval (CI) of 95\% was built. The $95 \%$ CI was then used to identify the frequencies in which the difference between both cohorts is significant.

Experimental design and statistical analyses. Statistical analysis was performed with GraphPad Prism version 8.00 (GraphPad Software) or IBM SPSS Statistics version 22 (IBM). Only values of $p<0.05$ were considered statistically significant. Normal distribution was evaluated in all datasets using the D'Agostino and Pearson, Kolmogorov-Smirnov, or Shapiro-Wilk normality tests when indicated. If the data fulfilled the criteria for normal distribution, we used the two-tailed unpaired or paired $t$ test. If the data did not pass the normality test, we used the Mann-Whitney test. Whenever multiple tests were performed on the same dataset, we adjusted our $p$ values using the Bonferroni correction procedure. An ANOVA (two-way ANOVA), and Bonferroni post hoc comparisons in case of significant interactions, was used to compare the data in Figure $2 E-G$. For cumulative distribution functions $(\mathrm{CDFs})$, the KolmogorovSmirnov test was used.

Linear mixed-effects models and generalized estimating equations. To assess differences between PV-Cre and PV-Cre/NR1f/f mice considering the subject variability, the variable of interest (state duration, modulation index, or power) was calculated at three different time points of the electrophysiological recordings, and the effects of Group (PV-Cre vs $\mathrm{PV}-\mathrm{Cre} / \mathrm{NR} 1 \mathrm{f} / \mathrm{f}$ ) and Time were assessed through a linear mixed-effects 
A

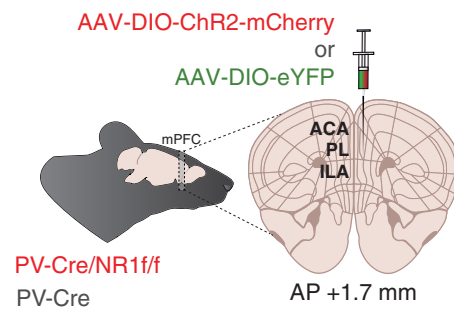

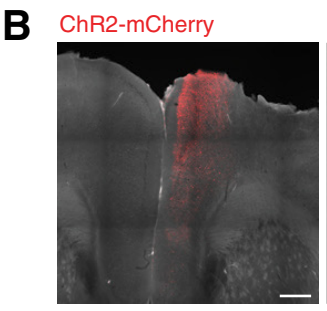

$\mathrm{AP}+1.44$

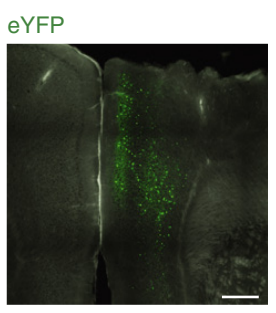

$\mathrm{AP}+1.56$

C PV-Cre/NR1f/f + AAV-DIO-ChR2-mCherry $(n=3)$
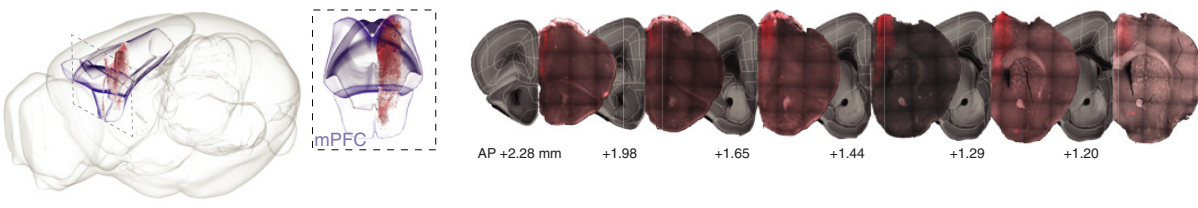

PV-Cre + AAV-DIO-ChR2-mCherry $(n=4)$
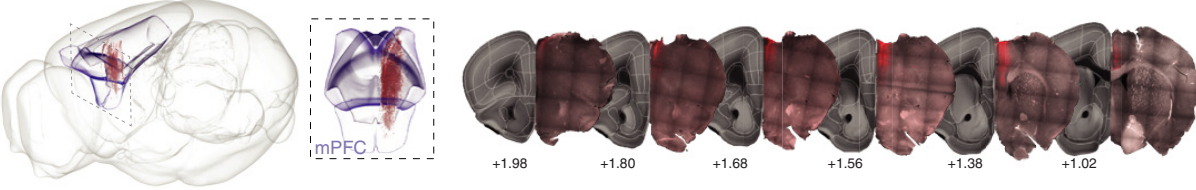

PV-Cre + AAV-DIO-eYFP $(n=4)$
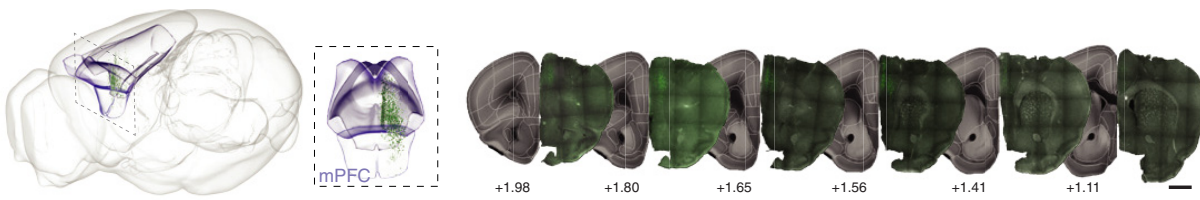

D
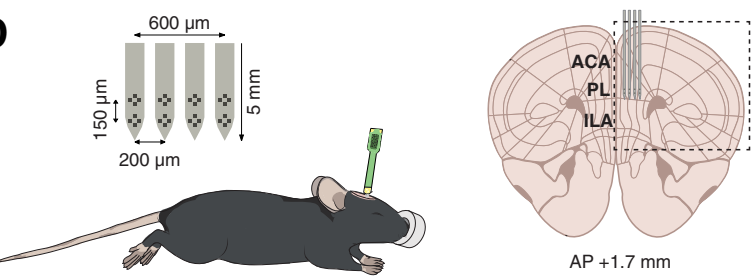

E

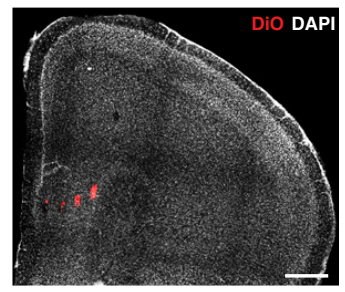

Figure 1. Virus expression and position of silicon probes. A, Illustration of viral injections. For optogenetic targeting of mPFC PV interneurons, AAV5-DI0-ChR2-mCherry (red) was unilaterally injected into the mPFC of PV-Cre/NR1f/f $(n=3)$ and PV-Cre $(n=4)$ mice. AAV5-DI0-eYFP (green) was unilaterally injected into the mPFC of PV-Cre $(n=4)$ for control of optogenetic artifacts (see Fig. 6E-G). B, Coronal sections of the mPFC showing expression of ChR2-mCherry (red; left) in a representative PV-Cre/NR1f/f mouse, and eYFP (green; right) in a representative PV-Cre mouse. C, Left, Representative 3D illustration of detected mPFC expression of ChR2-mCherry (top: PV-Cre/NR1f/f mouse; middle: PV-Cre mouse) and eYFP (bottom: PV-Cre mouse) registered to the Allen Mouse Brain Common Coordinate Framework version 3 (ABA_v3) reference atlas. Box represents the mPFC (ACA, ILA, and PL according to ABA_V3). Middle, Closeup (front view) of the box on the left. Right, Representative coronal sections used for detection and 3D plotting of virus-expressing neurons. $\boldsymbol{D}$, Illustration of the experimental setup. Electrophysiological recordings were conducted using a silicon probe in urethane-anesthetized mice. The four-shank eight-tetrode silicon probe was targeted to the PL area in the mPFC. $E$, Example Di0 (red) labeling of the four shank tracts of a silicon probe. Nuclei are counterstained by DAPI (white). ACA, Anterior cingulate area; IL, Infralimbic area. Scale bars: $\boldsymbol{B}, 300 \mu \mathrm{m} ; \boldsymbol{C}, 1 \mathrm{~mm} ; \boldsymbol{E}, 500 \mu \mathrm{m}$. Brain silhouette in $\boldsymbol{A}$ and mouse in $\boldsymbol{D}$ were sourced from https://scidraw.io/ and adapted from Kennedy (2020) and Tyler and Kravitz (2020), respectively.

model (LMM), with subject information assigned as random effects, and Group and Time as fixed effects. All models were estimated by the maximum likelihood and unstructured covariance, and the normality of residuals was verified. When models did not fit the LMM criteria (normality of residuals), Generalized Estimating Equations (GEE) model with a $\gamma$ regression was calculated, assigning Group and Time as fixed effects and the subject information as a random effect. Models were estimated by the maximum likelihood.

Bootstrap analysis. To overcome the different number of samples between individuals, a bootstrap procedure with 5000 iterations was used to evaluate significant differences of the variable of interest (state duration, modulation index, or power) between PV-Cre and PV-CreNR1f/f mice. A fixed number of events (equal to the smallest sample number between individuals) was randomly sampled from each individual (with reposition); and for each iteration, the group average was calculated. For the comparison, mean values were then transformed as the ratio from the PV-Cre group. The $95 \%$ CI was then used to identify significant differences between groups (CIs that did not include 1 were considered significantly different).

\section{Results}

To establish how dysfunction of PV inhibition affects synchrony in cortical networks, mice lacking NMDAR activity in PV neurons were used. Deletion of the NMDAR subunit NR1 ablates all NMDAR activity in the affected neuron (Forrest et al., 1994), and studies in transgenic mice have shown that NR1 deletion in PV neurons leads to cognitive dysfunction and aberrations in cortical network activity (Carlén et al., 2012; Hudson et al., 2020; for review, see Ju and Cui, 2016). Mice expressing Cre recombinase in PV neurons (PV-Cre mice) were crossed with mice carrying floxed alleles of the NR1 subunit (NR1f/f mice), generating $\mathrm{PV}-\mathrm{Cre} / \mathrm{NR} 1 \mathrm{f} / \mathrm{f}$ mice. PV-Cre mice were used as controls. To 

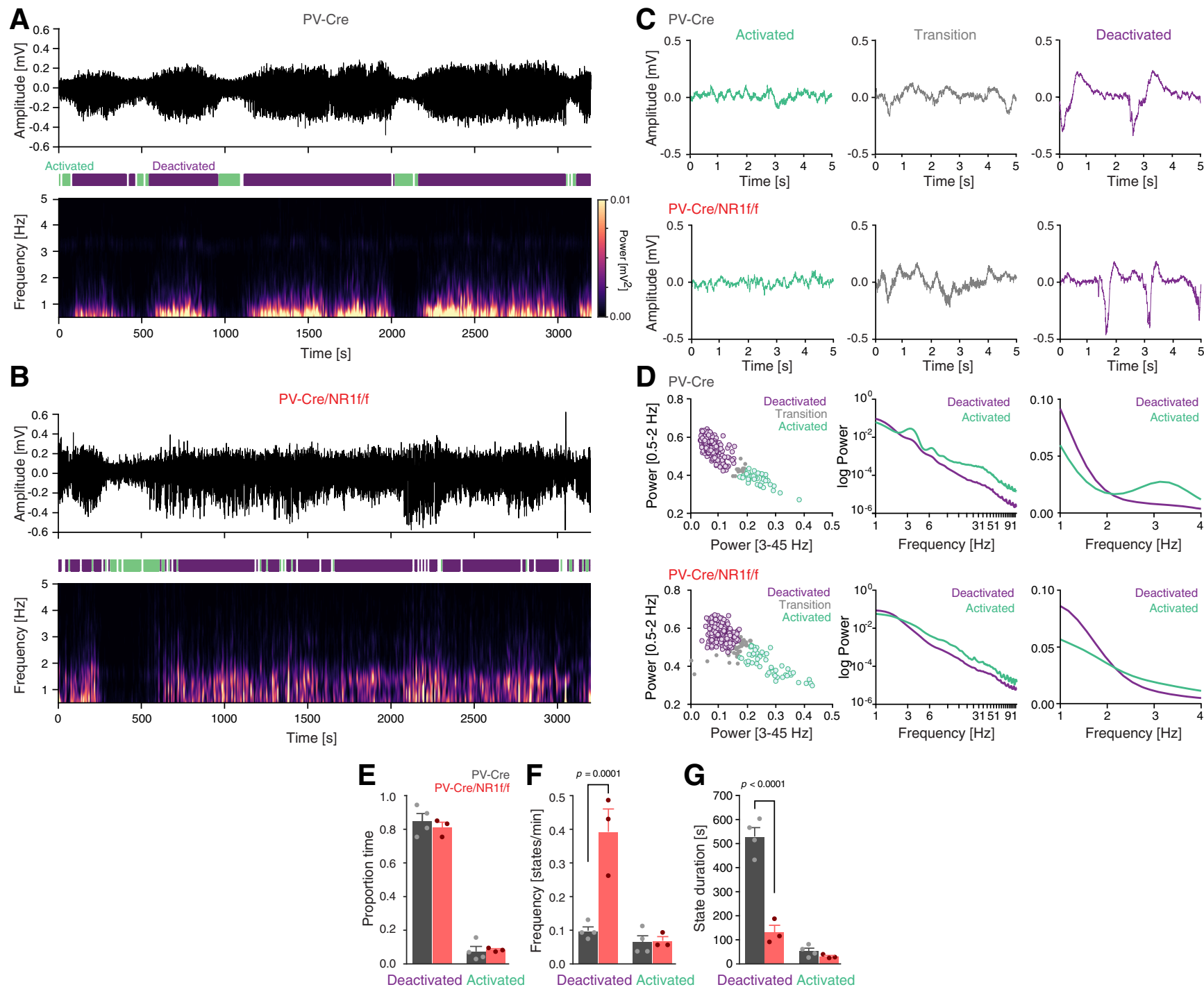

Figure 2. Mice with lack of NMDAR activity in PV neurons display altered cortical states. $\boldsymbol{A}, \boldsymbol{B}$, Representative mPFC LFP oscillations (3200 s) recorded under urethane anesthesia in a PV-Cre $(\boldsymbol{A})$ and a PV-Cre/NR1f/f (B) mouse, respectively. Top, Raw LFP. Middle, Color-coded outline of detected activated (green), deactivated (purple), and transition states (white). Bottom, Spectrogram (0-5 Hz) of the LFP trace in the top panel. $A$, Mice with intact NMDAR activity in PV neurons (PV-Cre) display typical cyclic transitions between deactivated (high-amplitude low-frequency [0.5-2 Hz] oscillations) and activated states (low-amplitude higher-frequency oscillations). $\boldsymbol{B}$, PV-Cre/NR1f/f mice show less defined slow oscillations along with altered transition patterns between deactivated and activated states. C, Representative LFP traces ( $5 \mathrm{~s}$ ) of activated (green), transition (gray), and deactivated states (purple), respectively, from a PV-Cre mouse (top) and a PV-Cre/NR1f/f mouse (bottom). D-G, PV-Cre mice ( $n=4$, gray) and PV-Cre/NR1f/f mice $(n=3$, red). D, Classification of brain states in PV-Cre mice (top) and PV-Cre/NR1f/f mice (bottom). Left, For detection of deactivated, activated, and transition (unclassified) states, a GMM was used for clustering of 30 s epochs (colored circles) of the LFP trace based on the power in the $0.5-2 \mathrm{~Hz}$ and 3-45 Hz frequency bands. Middle, Relative PSD (1-91 Hz) of the LFP for the activated (green) and deactivated (purple) states. Right, Magnification of the relative PSD of the LFP in the 1-4 Hz frequency band. $E-G$, Comparisons of the proportion (of total time), frequency (states/min), and duration, of deactivated and activated states in PV-Cre mice and PV-Cre/NR1f/f mice. $E$, There is no difference in the proportion time spent in deactivated and in activated states between PV-Cre/NR1f/f and PV-Cre mice $\left(F_{(1,10)}=0.1908, p=0.6715\right)$. Deactivated: PV-Cre: $0.85 \pm 0.04 ;$ PV-Cre/NR1f/f: 0.81 $\pm 0.03 ; p=0.8768 ;$ activated: PV-Cre: $0.07 \pm 0.03 ;$ PV-Cre/NR1f/f: $0.08 \pm 0.01 ; p>0.9999 . F$, Mice lacking NMDAR in PV neurons (PV-Cre/NR1f/f) transition into deactivated states significantly more often than mice with intact NMDAR in PV neurons (PV-Cre mice) $\left(F_{(1,10)}=0.2252, p=0.0008\right)$. Frequency (states $\left./ \mathrm{min}\right)$ of deactivated: PV-Cre: $0.098 \pm 0.012$ states $/ \mathrm{min} ; \mathrm{PV}-\mathrm{Cre} / \mathrm{NR} 1 \mathrm{f} /$ f: $0.394 \pm 0.068$ states $/ \mathrm{min} ; p=0.0001$; activated: PV-Cre: $0.066 \pm 0.018$ states $/ \mathrm{min} ;$ PV-Cre/NR1f/f: $0.069 \pm 0.013 \mathrm{states} / \mathrm{min} ; p>0.9999$. G, The duration of the deactivated states is significantly shorter in mice lacking NMDAR in PV neurons (PV-Cre/NR1f/f) than in mice with intact NMDAR in PV neurons $\left(\mathrm{PV}\right.$-Cre) $\left(F_{(1,10)}=64.89, p<0.0001\right)$. Deactivated: PV-Cre: $530.46 \pm 37.31$ s; PV-Cre/NR1f/f: $130.63 \pm 29.09$ s; $p<0.0001$; activated: PV-Cre: $51.88 \pm 11.79$ s; PV-Cre/NR1f/f: $29.33 \pm 4.44$ s; $p>0.9999$. Data are mean \pm SEM. $\boldsymbol{E}-\boldsymbol{G}$, Two-way ANOVA was used to assess significance, followed by a Bonferroni's multiple comparisons test if the ANOVA comparisons reached significance.

allow for light-activation and identification of the activity of mPFC PV interneurons in the electrophysiological recordings (opto-tagging) (H. Kim et al., 2016, 2020), an adeno-associated virus (AAV) vector with Cre-dependent expression of channelrhodopsin-2 (ChR2) fused to mCherry (AAV-DIO-ChR2-mCherry) (Cardin et al., 2009) was unilaterally targeted to the mPFC (PVCre/NR1f/f, $n=3$, PV-Cre, $n=4$; Fig. $1 A-C$ ). To control for any optogenetics artifacts, a cohort of 4 PV-Cre mice were injected with an AAV with Cre-dependent expression of the fluorophore eYFP (AAV-DIO-eYFP; Fig. 1A-C). These 4 PV-Cre mice were only used as controls in the opto-tagging experiments, and not used in any other analyses. Electrophysiological LFP recordings were conducted under urethane anesthesia 5-7 weeks after viral injections using a silicon probe (four shanks with two tetrodes 
each) targeted to the PL area in the mPFC (Fig. 1D,E). Urethane anesthesia is a validated model for testing mechanistic hypotheses regarding sleep-like brain oscillations (Hauer et al., 2019) and has been particularly used for the investigation of the effects of NMDAR antagonists on oscillatory patterns in different brain circuits (Kiss et al., 2013; Lopes-Aguiar et al., 2020). The use of an anesthetized preparation circumvents the increases in $\gamma$ power associated with hyperlocomotion elicited by administration of NMDAR antagonists in awake mice (Hakami et al., 2009).

\section{Mice with lack of NMDAR activity in PV neurons display altered cortical states and oscillatory patterns}

Urethane anesthesia displays spontaneous fluctuations between a deactivated state, characterized by slow oscillations $(0.5-2 \mathrm{~Hz})$ that resembles NREM sleep, and an activated state, characterized by faster oscillations $(>3 \mathrm{~Hz})$, mirroring aspects of REM sleep (Pagliardini et al., 2013). For characterization of the state dynamics over time, we divided the continuous LFP recordings ( $\sim 50 \mathrm{~min}$ ) into $30 \mathrm{~s}$ epochs and integrated the power for the 0.5 $2 \mathrm{~Hz}$ and $3-45 \mathrm{~Hz}$ frequency bands, respectively, for each epoch. Deactivated and activated brain states were classified using a GMM clustering approach using the power of the $0.5-2 \mathrm{~Hz}$ and $3-45 \mathrm{~Hz}$ bands (Fig. $2 A-D$ ). The mPFC activity in PV-Cre mice displayed typical rhythmic transitions between activated and deactivated states (Fig. $2 A$ ), with the activated state being characterized by high power in the $3-45 \mathrm{~Hz}$ frequency band and the deactivated state by high power in the slow oscillation bandwidth $(0.5-2 \mathrm{~Hz})$ (Fig. 2D). The strong presence of slow oscillations, specifically during deactivated states, can be observed in the spectrogram using a short-time Fourier transform (Fig. 2A). In between the two types of states, a transition period was identified, which exhibited intermediate electrographic characteristics (Fig. 2C). These short transition periods were not further analyzed. While deactivated and activated states were present and alternated, in PV-Cre/NR1f/f mice (Fig. 2B,C), the lack of NMDAR activity in PV neurons affected the state dynamics. The proportion of time spent in deactivated versus activated states, respectively, did not differ between PV-Cre/NR1f/f and PV-Cre mice. However, the deactivated states were significantly more frequent in PV-Cre/NR1f/f mice than in PV-Cre mice (Fig. 2E,F) and, thus, had a significantly shorter mean duration than the deactivated states in PV-Cre mice (Fig. 2G). No apparent difference in either frequency or duration of the activated states was found between PV-Cre/NR1f/f and PV-Cre mice (Fig. 2E-G). Of interest, sleep fragmentation has mainly been observed during NREM stages in both schizophrenia patients and animal models (Chouinard et al., 2004; Phillips et al., 2012).

Consistent with the literature (Carlén et al., 2012; Jadi et al., 2016), lack of NMDAR activity in PV neurons resulted in increased LFP power over a broad frequency spectrum (Fig. $3 A$ ). This increase was explicitly observed during the deactivated state and particularly pronounced for the mPFC HFB, which showed a significant difference between PV-Cre and PV-Cre/NR1f/f mice (Fig. 3B). No significant power differences between PV-Cre and PV-Cre/NR1f/f mice were detected in any frequency band $(0.5-150 \mathrm{~Hz})$ during activated states (data not shown). Different phases of low-frequency LFP oscillations can modulate faster oscillations $(>30 \mathrm{~Hz})$, an interaction suggested to regulate the spike probability and integrate activity across different temporal scales (Canolty and Knight, 2010). Therefore, we investigated the modulation of faster oscillations by the phase of slower oscillations during the deactivated states. For this, we built phase-amplitude comodulation maps (comodulograms) (Tort et al., 2010), which identified that the amplitude of the broadband $\gamma$ and the HFB in PV-Cre mice was most strongly modulated by the phase of $0.5-2 \mathrm{~Hz}$ (within the $\delta$ band) oscillations (Fig. 3C,D). Comparison of the MI revealed that the amplitude of both gamma and HFB was significantly less modulated by the slow oscillation in the PVCre/NR1f/f mice than in the PV-Cre mice (Fig. $3 E-G$ ). Importantly, the difference in modulation was not a result of differences in the power in the $0.5-2 \mathrm{~Hz}$ range between PV-Cre and PVCre/NR1 mice ( $U=9, p=0.4$, Mann-Whitney test). Together, the results suggest that intact NMDAR activity in PV neurons is required for proper temporal coordination of cortical network activity at multiple timescales, including coordination of cortical states (activated-deactivated states; minutes) and coordination between faster and slower oscillations of the LFP (seconds).

\section{Mice with lack of NMDAR activity in PV neurons display altered DOWN and UP states}

Under urethane, the deactivated brain state is dominated by slow oscillations, constituted by cyclic alternations between DOWN and UP states. During DOWN states, a marked period of populational hyperpolarization can be observed, while during UP states, cortical neurons are active (Steriade et al., 1993). Cortical DOWN and UP state dynamics can be quantified with simultaneous LFP and single-unit activity recordings (Saleem et al., $2010)$. We analyzed the mean firing rate ( $z$-scored) of single-unit activity during the deactivated states to detect periods of silence (DOWN) and activity (UP) (Fig. 4A). Plotting of the LFP spectrogram over time revealed that the UP states were marked by pronounced power at frequencies $>30 \mathrm{~Hz}$ in $\mathrm{PV}-\mathrm{Cre} / \mathrm{NR} 1 \mathrm{f} / \mathrm{f}$ mice, but not in PV-Cre mice (Fig. $4 A, B$ ). In addition, compared with PV-Cre mice, the DOWN state in PV-Cre/NR1f/f was marked by increased power in a broad HFB (including $\gamma$ frequencies; Fig. $4 A, B)$. Decomposing the LFP signal using the Morlet wavelet transform also illustrates the alterations in mice lacking NMDAR activity in PV neurons (Fig. 4C). Detailed analysis of the LFP during the DOWN-to-UP state transitions revealed that loss of NMDAR in PV neurons was associated with significantly reduced power at slower frequencies $(0.5-10 \mathrm{~Hz}$, Fig. $4 D, E)$. Separate analysis of DOWN and UP states showed enhanced power specifically in the $30-60 \mathrm{~Hz}$ band (i.e., within the $\gamma$ range) and in the $100-150 \mathrm{~Hz}$ (HFB) band during both states (Fig. $4 D, F, G$ ). The increased $\gamma$ and HFB power could contribute to the reduced phase-amplitude coupling during deactivated states in PV-Cre/NR1f/f mice (Fig. $3 C-G$ ). Analysis of the transition-triggered average LFP revealed a less prominent DOWN state in PV-Cre/NR1f/f mice, compared with PV-Cre mice (Fig. 4H), indicative of reduced hyperpolarization in the DOWN state of the slow oscillation (Saleem et al., 2010) in mice lacking NMDAR activity in PV neurons. The reduced hyperpolarization during DOWN states could contribute to the decreased power in lower LFP frequencies in PV-Cre/NR1f/f mice (Fig. $4 E$ ). The altered LFP activities in PV-Cre/NR1f/f mice were associated with significantly increased variability of the DOWN state duration (Fig. 4I). Further, PV-Cre/NR1f/f mice displayed significantly more UP state events with a shorter duration than PV-Cre mice (Fig. 4J), suggesting that the lack of NMDAR in PV neurons impacts the transition between DOWN and UP states.

\section{Mice with lack of NMDAR activity in PV neurons display disorganized oscillatory activity during DOWN and UP states}

As our results indicate increased $30-60 \mathrm{~Hz} \gamma$ and HFB power in PV-Cre/NR1f/f mice during DOWN and UP states (Fig. $4 F, G$ ), 
A

Deactivated
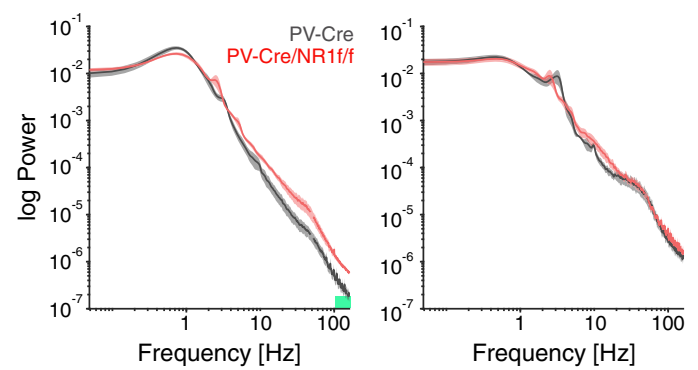

C

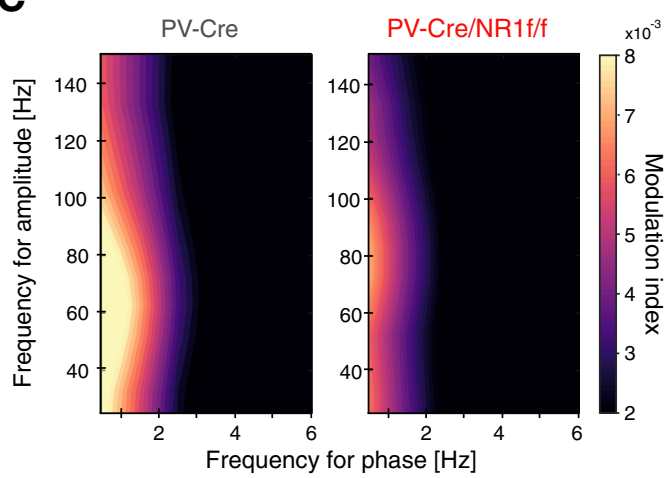

$\mathbf{E}$

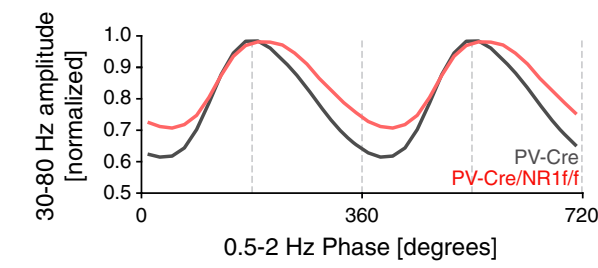

B Deactivated

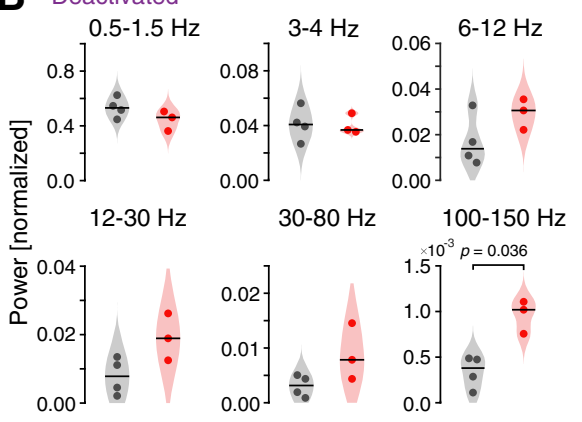

D
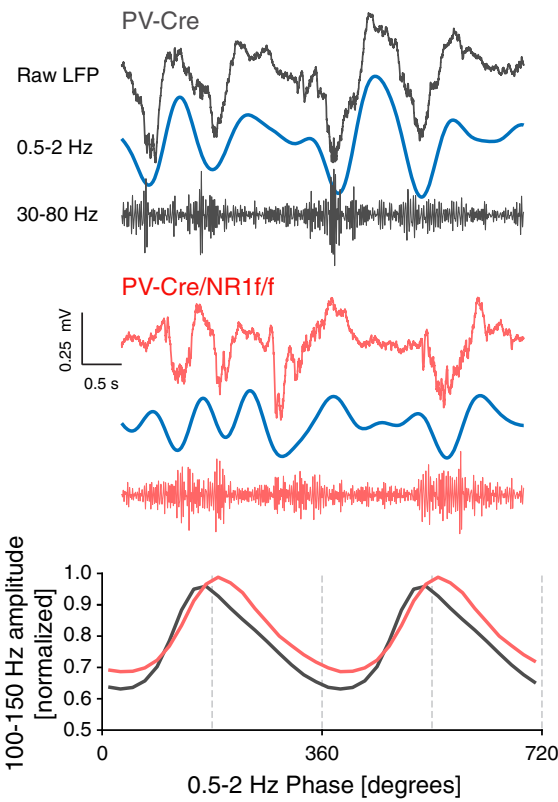
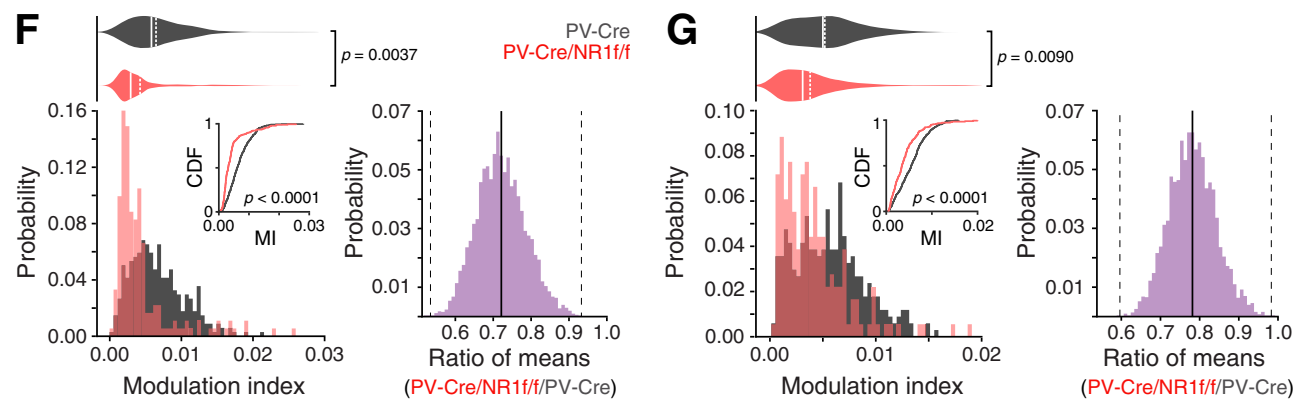

Figure 3. Mice with lack of NMDAR activity in PV neurons display increased broadband $\gamma$ power and decreased $\delta$-phase modulation of broadband $\gamma$ and HFB amplitude. A, B, E-G, PV-Cre mice $(n=4$, gray) and PV-Cre/NR1f/f mice ( $n=3$, red). $\boldsymbol{A}$, Mean PSD of the LFP of deactivated (left) and activated (right) states. Green bar represents frequency band (100-150 Hz) with significant power difference between PV-Cre and PV-Cre/NR1f/f mice. B, Comparisons of the integrated power at different frequency bands during deactivated states between PV-Cre and PV-Cre/ NR1f/f mice. Low- $\delta$ (0.5-1.5 Hz): PV-Cre: $0.53 \pm 0.04 ;$ PV-Cre/NR1f/f: $0.44 \pm 0.01 ; t=1.62, p=0.99 ;$ high- $\delta$ (3-4 Hz): PV-Cre: $0.041 \pm 0.006 ;$ PV-Cre/NR1f/f: $0.040 \pm 0.004 ; t=0.09, p=1 ;$ theta (6-12 Hz): PV-Cre: $0.017 \pm 0.006$; PV-Cre/NR1f/f: $0.029 \pm 0.004 ; t=-1.67, p=0.93 ; \beta$ (12-30 Hz): PV-Cre: $0.008 \pm 0.003 ;$ PV-Cre/NR1f/f: $0.019 \pm 0.004 ; t=-2.48, p=0.34 ;$ broadband $\gamma$ (30-80 Hz): PV-Cre: $0.003 \pm 0.001 ;$ PV-Cre/NR1f/f: $0.009 \pm 0.003 ; t=-2.12, p=0.52 ;$ HFB $(100-150 \mathrm{~Hz}):$ PV-Cre: $0.00034 \pm 0.0001 ;$ PV-Cre/NR1f/f: $0.00102 \pm 0.0001 ; t=$ $-4.55, p=0.036$. C, Representative phase-amplitude comodulograms of a PV-Cre (left) and a PV-Cre/NR1f/f mouse (right), respectively, showing the modulation of the broadband $\gamma$ and the HFB amplitude by the phase of $0.5-2 \mathrm{~Hz}$ oscillations. $\boldsymbol{D}$, Representative unfiltered LFP trace (top) and filtered $0.5-2 \mathrm{~Hz}$ (middle) and broadband $\gamma$ ( $30-80 \mathrm{~Hz}$; bottom) bands during a deactivated state in a PV-Cre and a PV-Cre/NR1f/f mouse, respectively. $\boldsymbol{E}$, Mean broadband $\gamma$ amplitude (left) and mean HFB amplitude (right) at different phases of the $0.5-2 \mathrm{~Hz}$ cycles. $\boldsymbol{F}$, Left, Comparison of the probability distribution of the MI between broadband $\gamma$ amplitude and $0.5-2 \mathrm{~Hz}$ phase shows that PV-Cre/NR1f/f mice have more deactivated epochs (30 s) with lower MI $(p<0.0001$, CDF inset) and significantly decreased comodulation between broadband $\gamma$ amplitude and $0.5-2 \mathrm{~Hz}$ phase compared with PV-Cre mice (PV-Cre: $0.0072 \pm 0.0012 ; \mathrm{PV}-\mathrm{Cre} / \mathrm{NR} 1 \mathrm{f} / \mathrm{f}$ : $0.0052 \pm 0.0021 ; F_{(1,7)}=18.180, p=0.0037$, LMM; right, Cl from bootstrap analysis: $\left.[0.50,0.92]\right) . G$, Left, Comparison of the probability distribution of the MI between HFB amplitude and $0.5-2 \mathrm{~Hz}$ phase shows that PV-Cre/NR1f/f mice have significantly more deactivated epochs (30 s) with lower MI $(p<0.0001$, CDF inset) and significantly decreased comodulation between HFB amplitude and $0.5-2 \mathrm{~Hz}$ phase compared with PV-Cre mice (PV-Cre: $0.0059 \pm 0.0014$; PV-Cre/NR1f/f: $0.0046 \pm 0.0013 ; F_{(1,7)}=12.826, p=0.0090$, LMM; right, Cl from bootstrap analysis: $[0.59,0.96])$. Data are mean \pm SEM. $\boldsymbol{A}$, Lines indicate mean. Shaded areas represent SEM. $\boldsymbol{B}$ (violin plots), Black line indicates median. Dot indicates mice. $\boldsymbol{F}, \boldsymbol{G}$ (violin plots), Solid line indicates mean. Dashed line indicates median. $\boldsymbol{F}, \boldsymbol{G}$ (bootstrap plots), Solid line indicates mean. Dashed line indicates $95 \%$ Cl. $\boldsymbol{B}$, Two-tailed unpaired $t$ test was used to assess significance after data passed the Shapiro-Wilk normality test, and Bonferroni adjustment was applied to correct for multiple comparisons. $\boldsymbol{F}, \boldsymbol{G}$, The Kolmogorov-Smirnov test was used to assess significance between CDFs, and complemented with an LMM and a bootstrap analysis to assess significance between PV-Cre and PV-Cre/NR1f/f mice while accounting for intragroup variability. Complementary statistical information can be found in Extended Data Figure 3-1. 


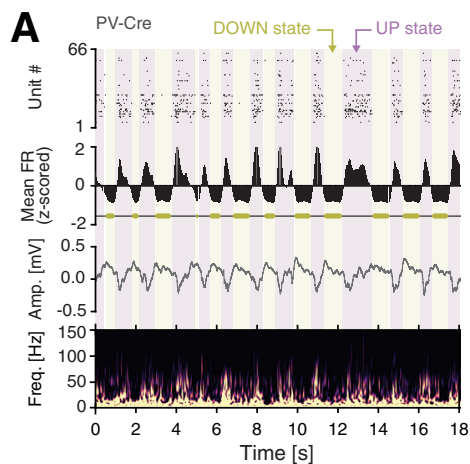

D
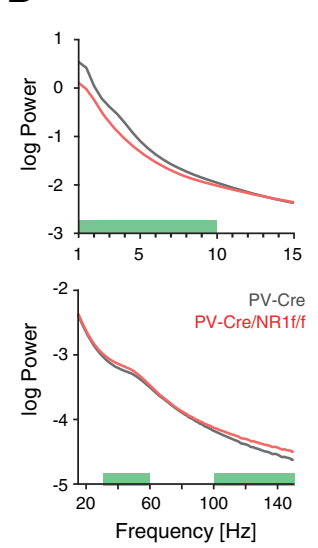

E
PV-Cre/NR1 f/f

$" 1$
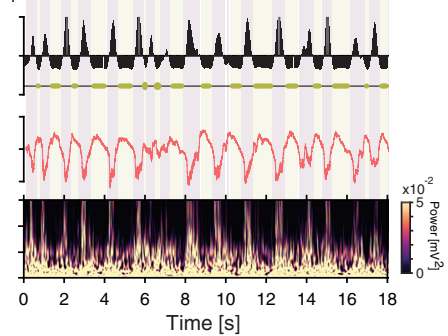

B

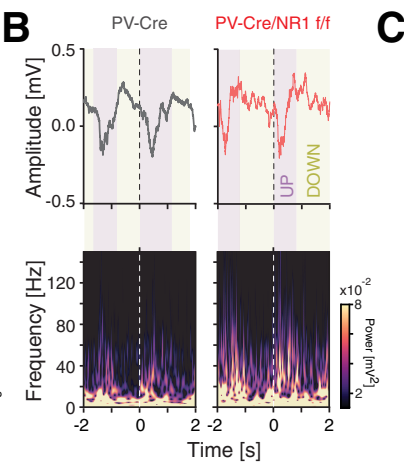

C

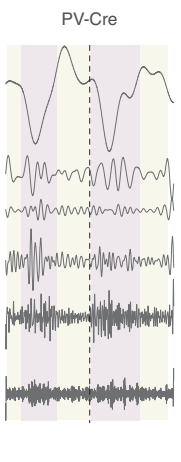

PV-Cre/NR1 f/f

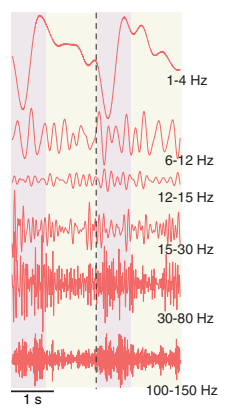

F

DOWN states
30-60 Hz

G UP states

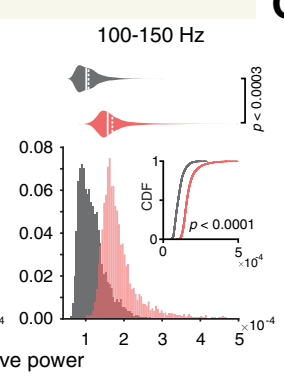

$30-60 \mathrm{~Hz}$

$100-150 \mathrm{~Hz}$

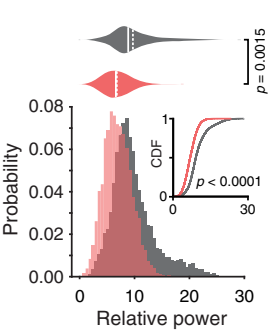

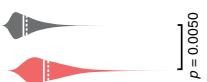
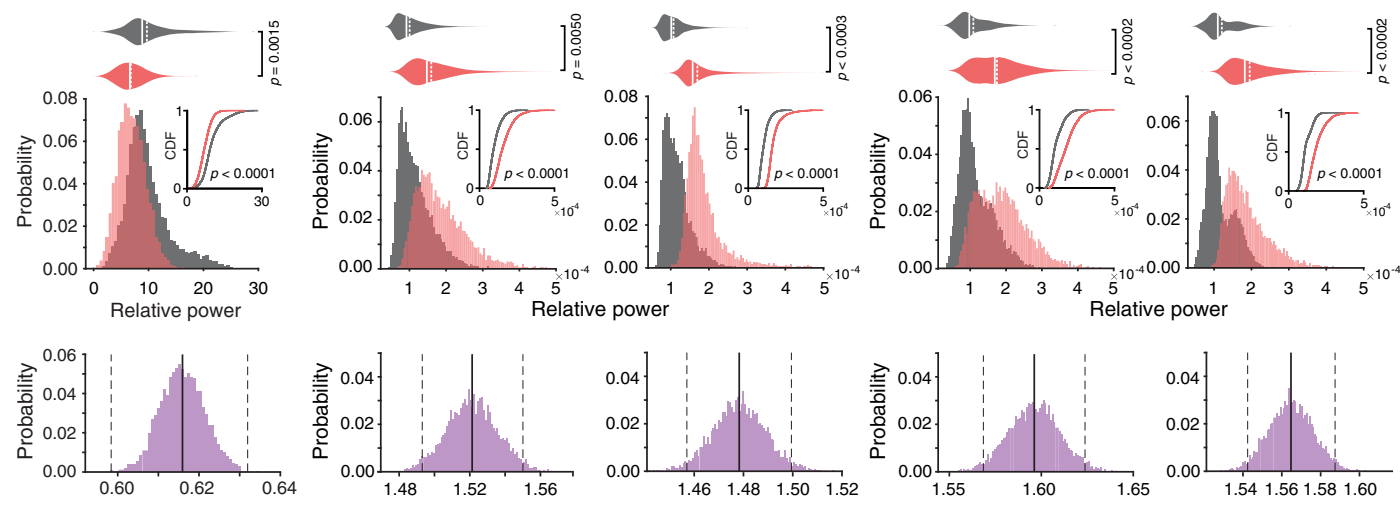

Ratio of means (PV-Cre/NR1f///PV-Cre)

H

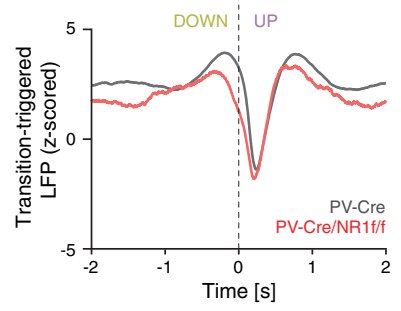

I DOWN states

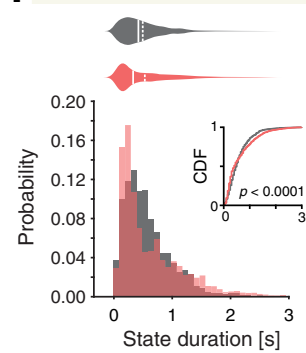

J UP states

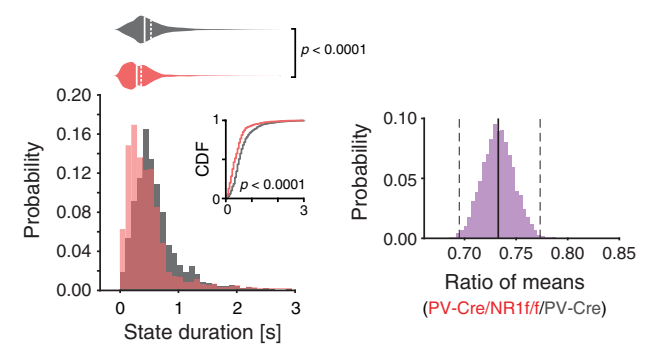

Figure 4. Mice with lack of NMDAR activity in PV neurons display altered LFP power during DOWN-to-UP state transitions and reduced duration of UP and DOWN states. $\boldsymbol{A}$, $\boldsymbol{B}$, Representative UP state (purple shading) and DOWN state (yellow shading) characteristics in a PV-Cre (gray) and a PV-Cre/NR1f/f (red) mouse, respectively. $\boldsymbol{A}$, From top to bottom, Raster plot of the recorded single units; the mean firing rate (FR, $z$-scored) with DOWN states represented with yellow horizontal bars; the raw LFP; spectrogram (0-150 Hz) of the LFP. Mice with intact NMDAR activity in PV neurons (PV-Cre) display typical cyclic transitions between periods of very low/no spiking activity (DOWN states) and high spiking activity (UP states). Mice lacking NMDAR in PV neurons (PV-Cre/NR1f/f mice) display shorter DOWN and UP states than mice with intact NMDAR in PV neurons. In addition, the UP states have increased LFP amplitude, particularly in LFP frequencies $>30 \mathrm{~Hz}$, in PV-Cre/NR1f/f mice. $\boldsymbol{B}$, Top, Representative unfiltered LFP traces (4 s) aligned to an example transition (time $=0 \mathrm{~s}$ ) from a DoWN state (yellow shading) to an UP state (purple shading) in a PV-Cre and a PV-Cre/NR1f/f mouse, respectively. Bottom, Spectrograms (0-150 Hz) of the LFP traces in the top panels, highlighting the marked power increase in higher frequencies $(>30 \mathrm{~Hz})$ during UP states. Mice with lack of NMDAR activity display increased power in higher frequencies $(>30 \mathrm{~Hz})$ during both DoWN and UP states. $C$, Representative Morlet wavelet filtering of the LFP traces in $\boldsymbol{B}$ at different frequency bands. $\boldsymbol{D}$-J, PV-Cre mice $(n=4$, gray) and PV-Cre/NR1f/f mice $(n=3$, red). $\boldsymbol{D}$, Comparison of the PSD of the LFP during the DOWN-to-UP state transition (-2 to $2 \mathrm{~s}$, see $\boldsymbol{H}$ ), between PV-Cre versus PV-Cre/NR1f/f mice. Green bars represent frequency bands with significant power difference between $\mathrm{PV}$-Cre and PV-Cre/NR1f/f mice. $\boldsymbol{E}$-G, Top, Comparison of the relative power in the frequency bands identified in $\boldsymbol{D}$ to hold differential power in PV-Cre versus PV-Cre/NR1f/f mice $(\boldsymbol{E}, 0.5-10 \mathrm{~Hz}$; $\boldsymbol{F}, \mathbf{G}, 30-60 \mathrm{~Hz}$ and $100-150 \mathrm{~Hz}$ ). $\boldsymbol{E}$, Comparison of the probability distributions of the relative power of the $0.5-10 \mathrm{~Hz}$ frequency band shows that PV-Cre/NR1f/f mice have more DOWN-to-UP state transitions with lower power $(p<0.0001$, CDF inset) and significantly decreased $0.5-10 \mathrm{~Hz}$ power during state transitions compared with PV-Cre mice (PV-Cre: $11.04 \pm 2.14$; PV-Cre/ NR1f/f: $6.76 \pm 0.35$; Wald $\chi^{2}=12.24, p=0.0015$, GEE; bottom, Cl from bootstrap analysis: [0.595, 0.632]). $\boldsymbol{F}$, Left, Top, Comparison of the probability distributions of the relative power of the $30-60 \mathrm{~Hz}$ frequency band shows that PV-Cre/NR1f/f mice have more DOWN states with high power in the 30-60 Hz band $(p<0.0001$, CDF inset) and significantly increased power in the $30-60 \mathrm{~Hz}$ band during DOWN states compared with PV-Cre (PV-Cre:1.25 $\times 10^{-4} \pm 0.15 \times 10^{-4}$; PV-Cre/NR1f/f: $1.90 \times 10^{-4} \pm 0.21 \times 10^{-4} ; F_{(1,7)}=198.8, p=0.0050$, LMM; bottom, Cl from bootstrap analysis: $[1.493,1.550])$. Right, Top, Comparison of the probability distributions of the relative power of the $100-150 \mathrm{~Hz}$ frequency band shows that PV-Cre/NR1f/f mice have more DOWN states with high power in the 100-150 Hz band ( $p<0.0001$, CDF inset) and significantly increased power in 100-150 Hz band during DoWN states compared with PV-Cre (PV-Cre: $1.24 \times 10^{-4} \pm 0.11 \times 10^{-4} ;$ PV-Cre/NR1f/f: $1.83 \times 10^{-4} \pm 0.06 \times 10^{-4} ; F_{(1,7)}=67.072, p=0.0003$, LMM; bottom, Cl from bootstrap analysis: [1.458, 1.499]). G, Left, Top, Comparison of the probability distributions of the relative power of the 30-60 Hz frequency band shows that PV-Cre/NR1f/f mice have more UP states with high power in the 30-60 Hz band $(p<0.0001$, CDF inset) and significantly increased power in the $30-60 \mathrm{~Hz}$ band during UP states compared with PV-Cre mice (PV-Cre: $1.26 \times 10^{-4} \pm 0.16 \times 10^{-4} ; \mathrm{PV}-$-Cre/NR1f/f: $2.01 \times 10^{-4} \pm 0.18$ $\times 10^{-4} ; F_{(1,7)}=197.338, p=0.0002, \mathrm{LMM}$; bottom, Cl from bootstrap analysis: $\left.[1.568,1.645]\right)$. Right, Top, Comparison of the probability distributions of the relative power of the 100-150 Hz frequency band shows that PV-Cre/NR1f/f mice have more UP states with high power in the $100-150 \mathrm{~Hz}$ band $(p<0.0001$, CDF inset) and significantly increased power in the $100-150 \mathrm{~Hz}$ band during UP states compared with PV-Cre mice (PV-Cre: $1.24 \times 10^{-4} \pm 0.14 \times 10^{-4} ;$ PV-Cre/NR1f/f: $1.94 \times 10^{-4} \pm 0.08 \times 10^{-4} ; F_{(1,7)}=77.880, p=0.0002$, LMM; bottom, Cl from bootstrap analysis: $[1.543,1.586])$. $\boldsymbol{H}$, Mean transition-triggered LFP traces (4s) of the DOWN-to-UP state transitions (time $=0 \mathrm{~s}$ ). $\boldsymbol{I}$, Left, Comparison of the probability distribution of the state 
we next sought to clarify whether this power increase was attributed to synchronous or asynchronous neuronal activity. Both highly synchronized neuronal activity and disorganized neuronal activity can increase the LFP power in different frequency bands, and PSD analysis is often used to determine the main oscillatory pattern emerging from the activity of a particular network in a specific moment. For instance, highly synchronized neuronal activity can lead to a stable buildup of LFP oscillations (i.e., a more sinusoidal-like oscillation), resulting in concentrated power in a narrow frequency band (e.g., sensory-evoked $\gamma$ oscillations), readily identified in the PSD. Disorganized neuronal activity generates a noisy and more irregular LFP, visible in the PSD as a more even power distribution over a broader frequency band (Uhlhaas et al., 2011). However, further analysis of the power spectrum is needed for quantitative comparison between different power distributions. To quantify the level of frequency dispersion (and consequently the level of synchronization in the neuronal activity) in the PSD, we performed analysis of the spectral entropy (based on Shannon entropy) of the LFP $(30-150 \mathrm{~Hz})$, where a narrower power distribution results in lower entropy values, and a large power distribution results in higher entropy values, with no dependency on the LFP frequency (Valero et al., 2017). Our analysis revealed that while the mean spectral entropy of the $30-150 \mathrm{~Hz}$ power spectrum in the UP and DOWN states did not differ between PV-Cre and PV-CreNR1f/f mice, the spectral entropy displayed significantly increased variability in the PV-Cre/NR1f/f mice (Fig. 5A,B). Of particular relevance, the $\mathrm{PV}-\mathrm{Cre} / \mathrm{NR} 1 \mathrm{f} / \mathrm{f}$ mice held more DOWN and UP events with high spectral entropy, indicating less synchronous LFP oscillations. In addition to the level of synchronization, the rate of neuronal firing can modify the power spectrum of the LFP through spectral leakage of spiking activity, where high spiking activity results in broadband increases in LFP power (Manning et al., 2009; Ray and Maunsell, 2011; Scheffer-Teixeira et al., 2013). LFP frequencies higher than $200 \mathrm{~Hz}$ have been suggested to reflect the presence of substantial spectral leakage (Belluscio et al., 2012). Thus, to quantify a contribution of spectral leakage caused by increased spiking activity to the LFP power in the 30$150 \mathrm{~Hz}$ frequency band, we calculated the power ratio between the $200-300 \mathrm{~Hz}$ and the $30-150 \mathrm{~Hz}$ bands, hereafter referred to as the HF index, for each DOWN and UP state event, respectively. Again, while the mean HF index of the UP and DOWN state events did not differ between PV-Cre and PV-CreNR1f/f mice, the $\mathrm{HF}$ index displayed significantly increased variability in the PV-Cre/NR1f/f mice (Fig. 5C,D). Particularly, the PV-Cre/NR1f/ f mice held more DOWN and UP state events with high HF index (i.e., with increased spectral leakage).

$\leftarrow$

duration of the DOWN states show that PV-Cre/NR1f/f mice have more DOWN states with long duration $(p<0.0001, \mathrm{CDF}$ inset). However, the mean DOWN state duration does not differ (PV-Cre: $0.63 \pm 0.06 \mathrm{~s}$; PV-Cre/NR1f/f: $0.69 \pm 0.06 s ; F_{(1,7)}=2.367, p=0.1680$, LMM). J, Left, Comparison of the probability distribution of the state duration of the UP states shows that PV-Cre/NR1f/f mice have more UP states with shorter duration $(p<0.0001$, CDF inset) and significantly decreased UP state duration compared with PV-Cre mice (PV-Cre: $0.62 \pm 0.03$; PV-Cre/NR1f/f: $0.45 \pm 0.03 ; F_{(1,6)}=223.11, p<0.0001$, LMM; right, Cl from bootstrap analysis: $[0.68,0.78]) . E-G, I, J$, The Kolmogorov-Smirnov test was used to assess significance between CDFs, and complemented with an LMM or GEE (in case of non-normality of residuals from LMM model) and a bootstrap analysis to assess significance between PV-Cre and PV-Cre/NR1f/f mice while accounting for intragroup variability. E-G, A Bonferroni adjustment was applied to correct for multiple comparisons. Complementary statistical information can be found in Extended Data Figure 4-1.
To infer the contribution of single-unit activity and activity in the frequency bands with differential power in PV-Cre/NR1f/f and PV-Cre mice $(30-60 \mathrm{~Hz}$, and $100-150 \mathrm{~Hz}$; Fig. $4 F, G)$ to the occurrence of high spectral entropy/high HF index events, we projected the average firing rate, the $30-60 \mathrm{~Hz}$ power, and 100 $150 \mathrm{~Hz}$ power, respectively, against the spectral entropy and the HF index. This identified that high spectral entropy/high HF index events (HF index $>1.96 \mathrm{SD}$ ) specifically in the PV-Cre/ NR1f/f mice were associated with increased firing rates during both DOWN and UP states (Fig. 5E,F). High spectral entropy/ high HF index events in PV-Cre/NR1f/f mice were furthermore associated with increased power in the HFB (100-150 Hz) (Fig. $5 G, H)$. In addition, high spectral entropy/high HF index was in the PV-Cre/NR1f/f mice associated with increased power in the $30-60 \mathrm{~Hz}$ band particularly during the UP states (Fig. 5I,J). Importantly, low spectral entropy/low HF index DOWN and UP state events were also associated with increased power in the 30$60 \mathrm{~Hz}$ band in both PV-Cre mice and PV-Cre/NR1f/f mice (Fig. $5 I, J)$. Thus, while PV-Cre and PV-Cre/NR1f/f mice shared events marked by increased $\gamma$ activity $(30-60 \mathrm{~Hz})$ (i.e., DOWN and UP state events with low spectral entropy, HF index, and firing rates), the PV-Cre/NR1f/f mice held high spectral entropy/high HF index UP states events with increased $30-60 \mathrm{~Hz}$ power. Direct investigation of the relationship between the power in 30$60 \mathrm{~Hz}$ and $100-150 \mathrm{~Hz}$ in events with high HF index (>1.96 SD) and firing rates confirmed a significant and strong positive correlation between the firing rate and the power of both the $\gamma$ band $(30-60 \mathrm{~Hz})$ and the HFB specifically in these events (Fig. $5 K, L$ ). This suggests that the increased power in the broadband $\gamma$ and HFB in the PV-Cre/NR1f/f mice is related to spectral leakage caused by increased firing rates associated with less synchronized LFP oscillations.

\section{Temporal dynamics of mPFC PV interneurons and excitatory neurons during DOWN-to-UP state transition}

Both cortical PV interneurons and excitatory neurons fire preferentially during the UP state (Puig et al., 2008; Tahvildari et al., 2012), but PV interneurons also display low firing activity during the DOWN state, which has been suggested to influence DOWN-to-UP transition probability (Zucca et al., 2017). As our LFP analyses indicated increased neuronal firing and altered state transitions in mice lacking NMDAR activity in PV neurons, our subsequent investigations focused on single-unit activities during DOWN-to-UP transitions. As before (H. Kim et al., 2016, 2020), we used opto-tagging to identify the activity of PV interneurons in PV-Cre and PV-Cre/NR1f/f mice injected with AAV-DIOChR2-mCherry (Fig. $1 A-C$ ). Application of blue light resulted in significantly increased firing in a set of neurons (Fig. 6A-C). Prefrontal inhibitory PV interneurons provide potent inhibition onto pyramidal neurons in the local network (H. Kim et al., 2016); and in agreement with this, the application of blue light resulted in the silencing of neurons in the local circuit (Fig. 6D). In contrast, the blue light application did not modulate prefrontal activities in PV-Cre mice injected with AAV-DIO-eYFP $(n=4$; Fig. $6 E-G)$. The spike waveforms of the light-activated units $(n=5$; Fig. $6 H-K)$ were signified by short half-valley width and low peak-to-valley amplitude ratio, typical of prefrontal NS interneurons (Fig. 7A) (H. Kim et al., 2016). These typical features were further used to classify recorded units into neuronal subtypes by calculating the NS probability in a GMM clustering (Fig. $7 B$ ). Based on the NS probability units were classified as WS putative pyramidal neurons $(n=155$, NS probability $<0.3)$ or NS putative interneurons $(n=63$, NS probability $>0.9)$, 

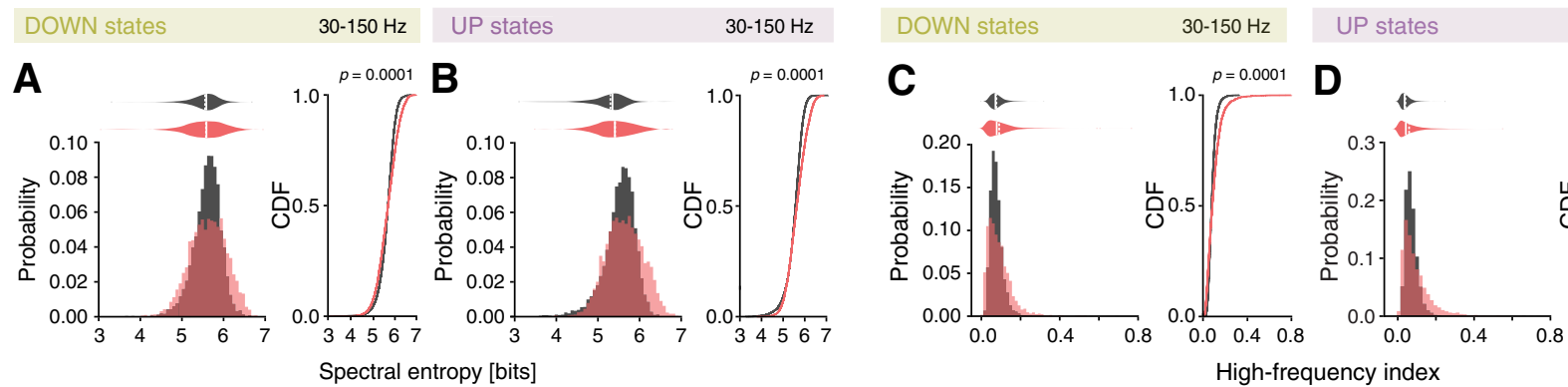

$30-150 \mathrm{~Hz}$

High-frequency index
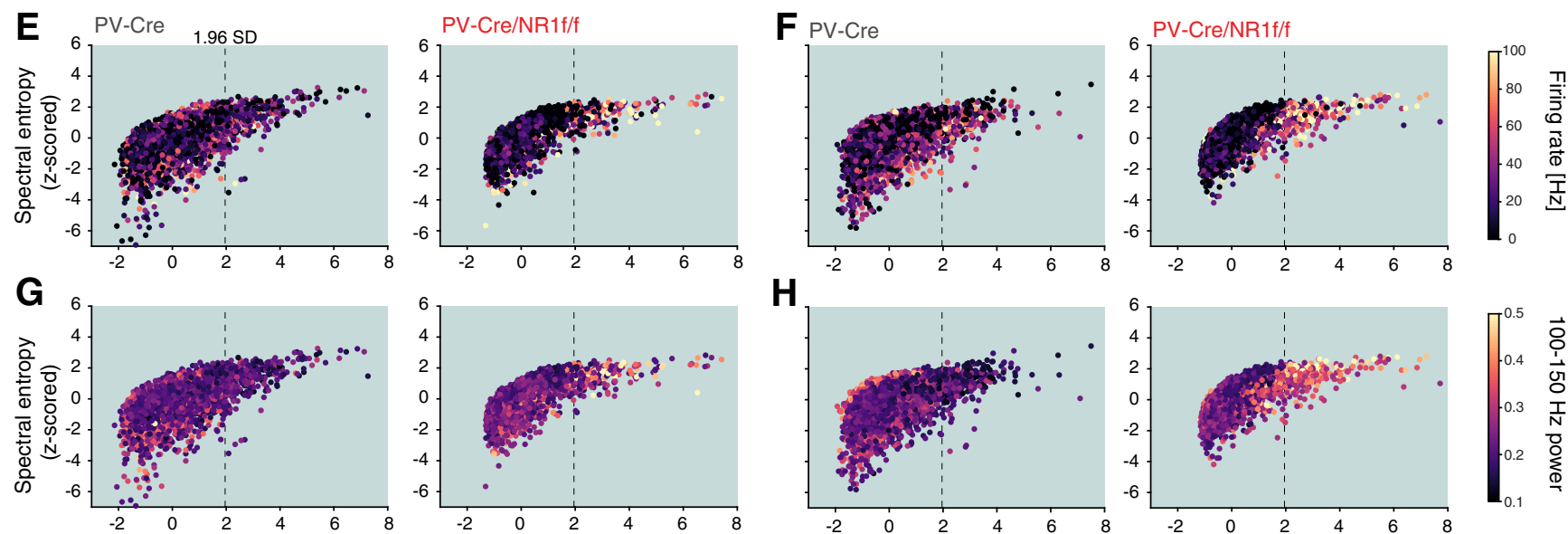

$\mathbf{H}$
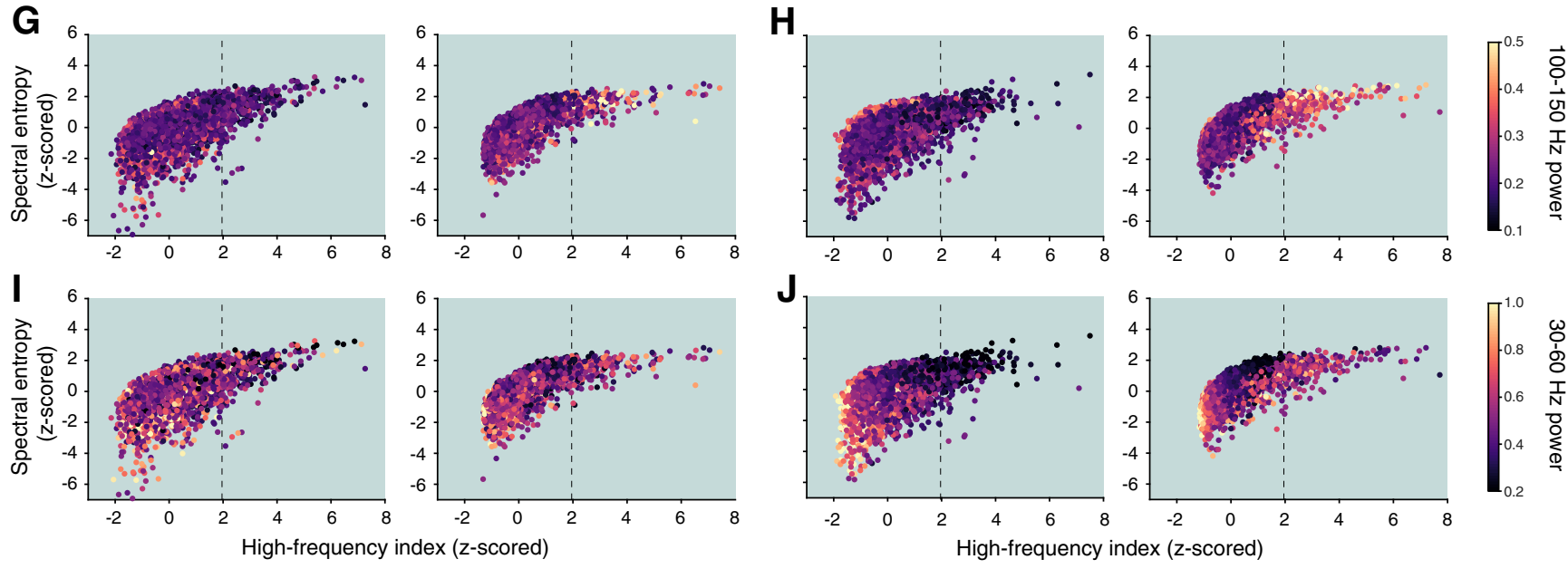

UP states

$30-150 \mathrm{~Hz}$

$\mathbf{K}$ High frequency index $<1.96 \mathrm{SD}$ (outside of the tail of the distribution)

L High frequency index > 1.96 SD (tail of the distribution)
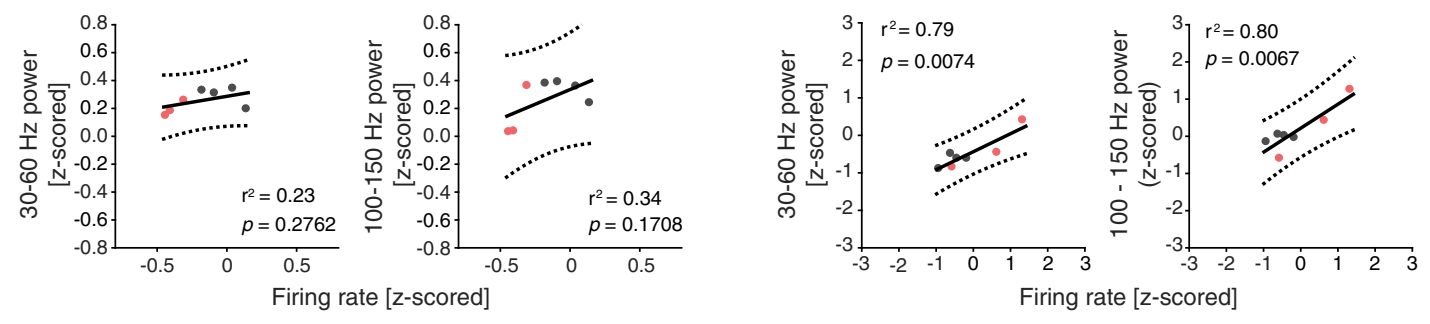

Figure 5. Mice with lack of NMDAR activity in PV neurons display asynchronous oscillatory activity associated with high firing rate and high-frequency $(>30 \mathrm{~Hz})$ power during DOWN and UP states. $A-L$, PV-Cre $(n=4$; gray) and PV-Cre/NR1f/f mice $(n=3$; red). $\boldsymbol{A}$, Left, Comparison of the probability distribution of the spectral entropy of high LFP frequencies (30-150 Hz) shows a significantly different distribution between PV-Cre and PV-Cre/NR1f/f mice $(p<0.0001$, CDF; right), with the PV-Cre/NR1f/f mice having more DOWN states with low and high spectral entropy, respectively, compared with PV-Cre mice. However, the mean spectral entropy does not differ (PV-Cre: $5.58 \pm 0.08$; PV-Cre/NR1f/f: $\left.5.57 \pm 0.15 ; F_{(1,7)}=0.005, p=0.9480, \mathrm{LMM}\right) . \boldsymbol{B}$, Left, Comparison of the probability distribution of the spectral entropy of high LFP frequencies $(30-150 \mathrm{~Hz})$ shows a significantly different distribution between PV-Cre and PV-Cre/NR1f/f mice $(p<0.0001$, CDF; right), with the PV-Cre/NR1f/f mice having more UP states with low and high spectral entropy, respectively, compared with PV-Cre mice. However, the mean spectral entropy does not differ (PV-Cre: $5.47 \pm 0.06$; PV-Cre/NR1f/f: $5.57 \pm 0.13 ; F_{(1,7)}=0.138, p=0.7220$, LMM). C, Left, Comparison of the probability distribution of the HF index (calculated from the power ratio: $200-300 \mathrm{~Hz} / 30-150 \mathrm{~Hz})$ shows a significantly different distribution between PV-Cre and PV-Cre/NR1f/f mice $(p<0.0001$, CDF; right), with the PV-Cre/NR1f/f mice having more DOWN states with low and high HF index, respectively, compared with PV-Cre mice. However, the mean HF index does not differ (PV-Cre: 0.076 \pm 0.010 ; PV-Cre/NR1f/f: 0.082 \pm 0.022 ; $F_{(1,7)}=0.490, p=0.5070$, LMM). $\boldsymbol{D}$, Left, Comparison of the probability distribution of the HF index shows a significantly different distribution between PV-Cre and PV-Cre/NR1f/f mice $(p<0.0001,(D F ;$ right), with the PV-Cre/NR1f/f mice having more UP states with low and high HF index, respectively, compared with PV-Cre mice. However, the mean HF index does not differ (PV-Cre: $0.065 \pm 0.001 ;$ PV-Cre/NR1f/f: $\left.0.082 \pm 0.024 ; F_{(1,7)}=0.819, p=0.3960, \mathrm{LMM}\right)$. $\boldsymbol{E}-\mathrm{J}$, Projection (as color maps) of the firing rate $(\boldsymbol{E}, \boldsymbol{F})$, power of $100-150 \mathrm{~Hz}$ LFP $(\boldsymbol{G}, \boldsymbol{H})$, and power of 30-60 Hz LFP (I, ) over spectral entropy (z-scored) versus HF index (z-scored) (PV-Cre: $n=9493$; PV-Cre/NR1f/f: $n=5971$ DOWN + UP events). The data points (dots) are randomly plotted. Dashed lines indicate $1.96 \mathrm{SD}$. The high spectral entropy/high HF index events are specifically in PV-Cre/NR1f/f mice associated with high firing rate and high-frequency ( $>30 \mathrm{~Hz}$ ) power. $\boldsymbol{E}, \boldsymbol{F}$, The high spectral entropy/high HF index events in PV-Cre/NR1f/f mice are marked by a high firing rate. $\mathbf{G}, \boldsymbol{H}$, The high spectral entropy/high HF index events in PV-Cre/NR1f/f mice are marked by high 100-150 Hz LFP power. I, J, Low spectral entropy/low HF index in both PV-Cre and PV-Cre/NR1f/f mice are marked by high 30-60 Hz (within $\gamma$ range) power. However, during UP 
respectively. Unclassified units ( $n=98$, NS probability between 0.3 and 0.9 ) were not further analyzed (Fig. 7C,D). A second GMM clustering based on peak distribution (calculated with normalized amplitude waveform) was applied to further classify all NS units ( $n=63$ units) as putative PV ( $n=14$ units, probability $>0.9)$ and NS ( $n=49$ units, probability $<0.3)$. This classification was further confirmed by the calculation of the spike waveform similarity index with light-induced spike waveforms, where the PV interneurons within the NS population presented a waveform similarity index $>95 \%$. (Fig. $7 E, F$ ). In summary, our analyses identified WS, NS, and putative PV interneurons, respectively, with distinct spike waveform features (Fig. $7 G$ ).

Isolation of $1 \mathrm{~s}$ windows around the DOWN-to-UP state transitions ( $0.5 \mathrm{~s}$ before and $0.5 \mathrm{~s}$ after state transition) and analysis of the mean firing rate ( $z$-scored) of individual mPFC WS, NS, and PV interneurons, respectively, revealed that all three classes of neurons displayed firing primarily in the UP state in both PV-Cre/NR1f/f and PV-Cre mice (Fig. 8A-I). While the mean WS firing rate during UP states was similar between PVCre/NR1f/f and PV-Cre mice (Fig. $8 B$ ), the spike time variability was significantly increased and the peak latency of the firing increased in PV-Cre/NR1f/f mice compared with PV-Cre mice (Fig. 8A,C). Furthermore, the average firing rate of WS neurons was significantly increased in PV-Cre/NR1f/f1 mice during DOWN states, compared with PV-Cre mice (Fig. $8 B$ ); and together, the data suggest reduced precision in the coordination of WS activities in mice lacking NMDAR activity in PV neurons. We found this aberration to be accompanied by a significantly increased peak latency of the PV firing during the UP states in PV-Cre/NR1f/f mice (Fig. 8D-F). The dynamics of NS putative interneurons during state transition was similar in PV-Cre/ NR1f/f and PV-Cre mice (Fig. 8G-I). Overall, the analyses of single-unit activities show impaired WS spike precision in mice with lack of NMDAR activity in PV interneurons, consistent with previous findings in the somatosensory cortex (Carlén et al., 2012), supporting the view that cortical PV interneurons are integral to the neuronal dynamics in the $\mathrm{MPFC}$ during state transitions (Massi et al., 2012; Zucca et al., 2017).

\section{Distinct characteristics of broadband $\gamma$ oscillations induced by ketamine}

Local mPFC administration of NMDAR antagonists is known to elicit marked alterations of oscillatory patterns, inducing a state with lower LFP amplitude and increased power in faster frequencies $(>30 \mathrm{~Hz}$ ) (Kulikova et al., 2012; Lee et al., 2017). We questioned whether the ketamine-induced oscillatory alterations are related to asynchronous neuronal activity (e.g., increased firing rate and/or decreased spike precision that we found to be

$\leftarrow$

states, PV-Cre/NR1f/f mice in addition display high spectral entropy/high HF index events marked by increased $30-60 \mathrm{~Hz}$ LFP power. $\boldsymbol{K}, \boldsymbol{L}$, Relationship between high-frequency $(>30 \mathrm{~Hz})$ power and the neuronal firing rate in UP state events with low $\mathrm{HF}$ index $(<1.96$ SD, i.e., the upper limit of the $95 \% \mathrm{Cl})(\boldsymbol{K})$, and UP states events with high $\mathrm{HF}$ index $(>1.96 \mathrm{SD})(\boldsymbol{L})$. There is a significant and strong positive correlation between the firing rate and the power of both the broadband $\gamma\left(30-60 \mathrm{~Hz} ; r^{2}=0.79, p=0.0071\right)$ and the HFB $\left(100-150 \mathrm{~Hz} ; r^{2}=0.81, p=0.0060\right)$ band in the events with $\mathrm{HF}$ index ( $\left.>1.96 \mathrm{SD}\right)$, but not in the events with low HF index $(<1.96 \mathrm{SD})$. Line indicates linear regression. Dashed line indicates $95 \% \mathrm{Cl}$. For the CDFs $(\boldsymbol{A}-\boldsymbol{D})$, the Kolmogorov-Smirnov test was used to assess significance, and complemented with an LMM analysis to assess significance between PV-Cre and PV-Cre/NR1f/f mice while accounting for intragroup variability. $\boldsymbol{K}, \boldsymbol{L}$, A linear regression was used, and a Pearson correlation coefficient was calculated. Complementary statistical information can be found in Extended Data Figure 5-1. associated with increased high-frequency power in PV-Cre/ NR1f/f mice). For this, the silicon probe was moved to a new $\mathrm{mPFC}$ recording site, and $30 \mathrm{~min}$ baseline single-unit and LFP oscillations were recorded. ( + -)-ketamine was thereafter locally applied into the mPFC (see Materials and Methods), and the effects were recorded for $30 \mathrm{~min}$ (60 min recording time in total; PV-Cre, $n=3$ mice; PV-Cre/NR1f/f; $n=3$ mice). In accordance with earlier reports (Kulikova et al., 2012), ketamine application triggered a rapid shift in the LFP oscillations in PV-Cre mice, from predominantly low frequencies of high-amplitude to a state with persistent high-frequency $(>30 \mathrm{~Hz})$ activity of low-amplitude (Fig. 9A-C). The LFP oscillations in the ketamine-induced state were desynchronized, as revealed by plotting of the autocorrelogram of the LFP over time (60 min) (Fig. 9D). In contrast, ketamine application did not overly affect the LFP oscillations in mice lacking NMDAR activity in PV neurons (Fig. 9A-D). Instead, PV-Cre/NR1f/f mice displayed low-frequency activity of high-amplitude both before and after application of ketamine (Fig. 9A, B). Ketamine increased the power of most frequency bands in PV-Cre mice (Fig. 9C), and the power change was significantly larger than in PV-Cre/NR1f/f mice (Fig. 9C). Consequently, PV-Cre mice displayed significantly higher broadband $\gamma$ and HFB power after local application of ketamine compared with PV-Cre/NR1f/f mice (Fig. 9E-G). In addition, the low $\delta(0.5-1.5 \mathrm{~Hz})$ power was significantly lower in PV-Cre mice than in PV-Cre/NR1f/f mice (Fig. 9E-G). Our results confirm that NMDAR in PV neurons are a central target of NMDAR antagonists (Homayoun and Moghaddam, 2007; Hakami et al., 2009; Carlén et al., 2012; Cohen et al., 2015; Jadi et al., 2016; Picard et al., 2019; Hudson et al., 2020), and show that PV interneurons are central to ketamine's effects on activities in prefrontal networks.

We furthermore questioned how ketamine-induced broadband $\gamma$ oscillations are coordinated by lower LFP frequencies. Based on our previous results, we focused on the coupling between the $0.5-2 \mathrm{~Hz}$ phase and the broadband $\gamma(30-80 \mathrm{~Hz}) \mathrm{am}-$ plitude, which we found to be significantly lower in PV-Cre/ $\mathrm{NR} 1 \mathrm{f} / \mathrm{f}$ mice than in PV-Cre mice during deactivated states, a reflection of desynchronized LFP oscillations in PV-Cre/NR1f/f mice (Fig. 3C-F). Interestingly, the $\delta-\gamma$ phase-amplitude coupling was significantly decreased in PV-Cre mice compared with PV-Cre/NR1f/f mice after ketamine application (Fig. 9H-K). The blunted response to ketamine in mice lacking NMDAR activity in PV neurons was also evident in the single-unit dynamics. While the ketamine state was characterized by a more tonic firing in PV-Cre mice, the single-unit activity in PV-Cre/NR1f/f mice after ketamine application remained oscillating between silent (DOWN) and active (UP) periods (Fig. 10A,B), with the mean firing rate of both WS neurons and NS interneurons (the latter including putative PV interneurons) being significantly higher in PV-Cre mice than in PV-Cre/NR1f/f mice (Fig. $10 C, D)$. Together, the analyses show that local ketamine application induced desynchronized LFP oscillations, increased firing rates, and increased power in a (very) broad range of LFP oscillations in mice with intact NMDAR activity in PV neurons. In addition, the coupling between broadband $\gamma$ amplitude and the phase of low-frequency oscillations decreased.

\section{Prefrontal spike-LFP entrainment during baseline and after ketamine application}

LFP oscillations contribute to spatiotemporal coordination of neuronal activity, modulating the spiking probability according to the phase and frequency of the field oscillations (Watson et al., 
2018). As we found (increased) spiking in $\mathrm{PV}-\mathrm{Cre} / \mathrm{NR} 1 \mathrm{f} / \mathrm{f}$ mice contaminating the LFP $>30 \mathrm{~Hz}$ (Fig. $5 E-J$ ), we, as a next step, analyzed the entrainment of spikes to the LFP with a specific focus on the broadband $\gamma(30-80 \mathrm{~Hz})$ and HFB. Using normal circular distribution statistics (see Materials and Methods), we first analyzed how the spiking of the different neuronal populations (WS, NS, and PV, respectively) was modulated by higher LFP frequencies (21-242 Hz) during baseline (030 min before ketamine application; Fig. $10 E-G)$. To measure the strength of the population entrainment, we calculated the proportion of neurons statistically $(p<0.01)$ modulated by each specific LFP frequency. During baseline, the spiking of both the WS and the PV neurons in the PV-Cre mice was most strongly entrained by higher LFP frequencies (Fig. $10 F)$. In contrast, the two populations were most strongly entrained by a lower and broader frequency spectrum in PVCre/NR1f/f mice (Fig. 10F). A bootstrap procedure (see Materials and Methods), used to detect significant differences in the LFP entrainment of spikes between PV-Cre/NR1f/f and PV-Cre mice, confirmed a significantly stronger spike entrainment by high-frequency LFP oscillations $(\sim 90-120 \mathrm{~Hz})$ in PV-Cre mice than in PV-Cre/NR1f/f mice (Fig. 10F). The spiking in PV-Cre/NR1f/f mice was significantly stronger, modulated by $<40 \mathrm{~Hz}$ oscillations than in PV-Cre mice (Fig. 10F).

We next investigated how the desynchronization induced by ketamine alters the LFP entrainment of spikes in the mPFC network and the plausible dependence on NMDAR activity in PV neurons. This revealed that ketamine remarkably reorganized the spike entrainment in mice with intact NMDAR activity in PV neurons. Specifically, the preferential modulation of the WS neurons shifted toward faster LFP frequencies $(>200 \mathrm{~Hz}$ ) (Fig. $10 G)$, while the PV interneurons were modulated by a broader range of LFP frequencies during the ketamine state than during baseline (Fig. 10G). In contrast, the altered entrainment of spikes to the LFP in PV-Cre/NR1f/f mice seen during baseline (Fig. 10F) at large persisted after application of ketamine (Fig. 10G), strongly indicating that $\mathrm{PV}$ interneurons (with intact NMDAR activity) are integral to proper LFP entrainment of spiking in the mPFC. Importantly, the LFP entrainment of spiking activity during the

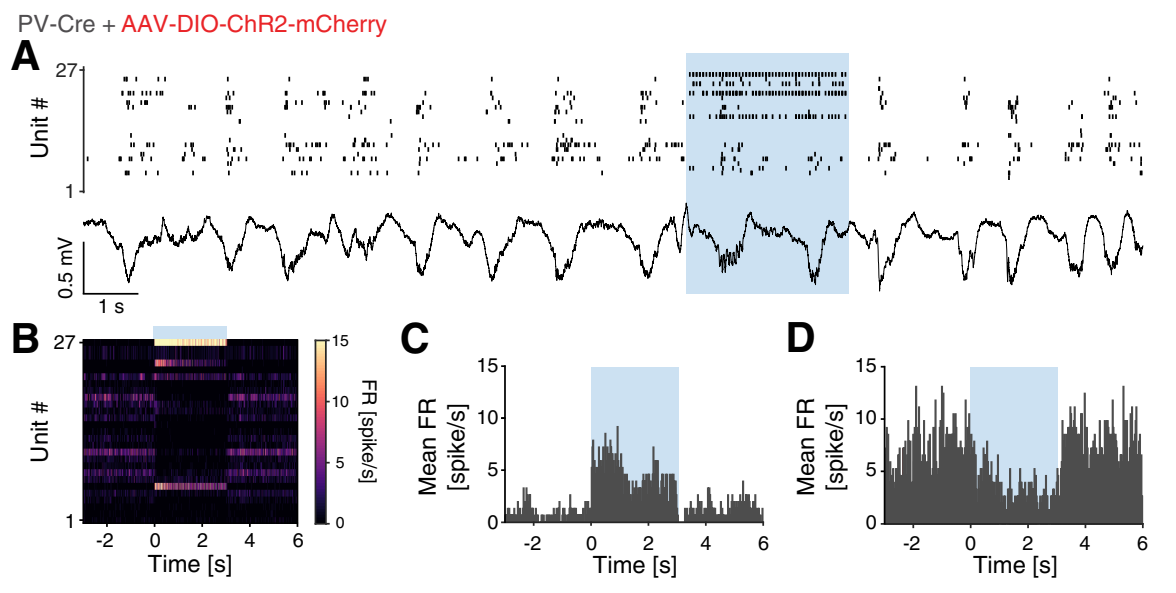

PV-Cre + AAV-DIO-eYFP
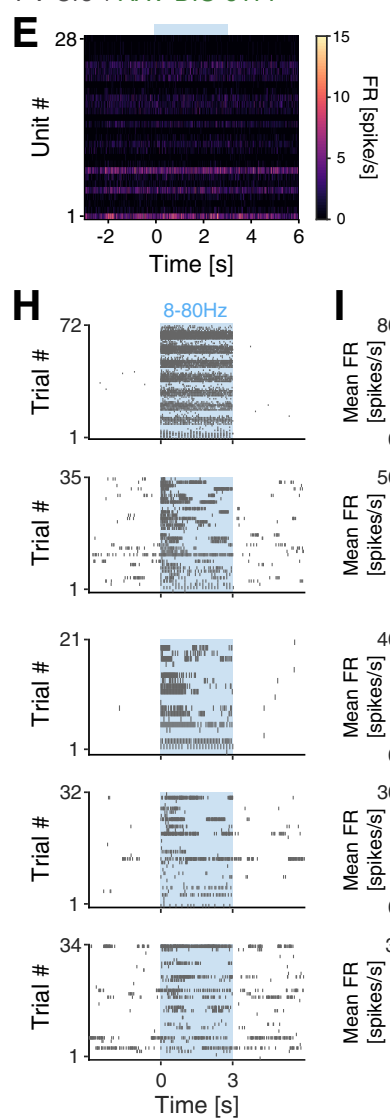

$\mathbf{F}$
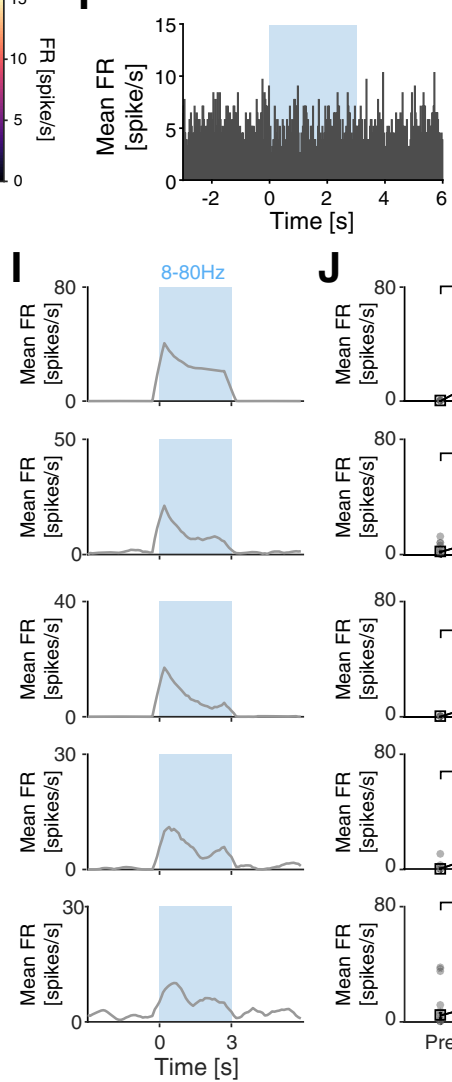
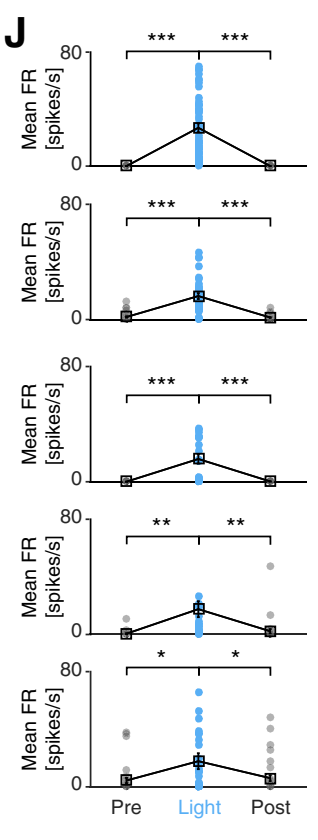

G

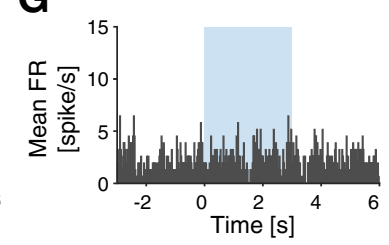

Figure 6. Opto-tagging of ChR2-expressing mPFC PV interneurons. $\boldsymbol{A}$, Example spiking activity of single units (top; $n=27$ ) and raw LFP (bottom) recorded (20 s) in the mPFC of a PV-Cre mouse injected with AAV-DI0-ChR2-mCherry. Blue light application (blue shading; $3 \mathrm{~s}, 473 \mathrm{~nm}, 46 \mathrm{~mW} / \mathrm{mm}^{2}, 3 \mathrm{~ms}$ pulses, $40 \mathrm{~Hz}$ ) modulates the activity of mPFC neurons. $\boldsymbol{B}$, Mean FR of the neurons in $\boldsymbol{A}$ across 90 trials ( $9 \mathrm{~s}$ peristimulus time-histogram [PSTH]). C, PSTH ( $9 \mathrm{~s}$ ) demonstrating increased FR of a light-activated unit in $\boldsymbol{A}$ in response to blue light application. $\boldsymbol{D}$, PSTH $(9 \mathrm{~s})$ demonstrating decreased FR of an mPFC unit in $\boldsymbol{A}$ in response to light activation of local PV interneurons. $\boldsymbol{E}$-G, Blue light application did not modulate the firing rates in PV-Cre mice injected with AAV-DI0-eYFP. Experimental settings as in $\boldsymbol{A}$. $\boldsymbol{E}$, Mean FR of mPFC neurons $(n=28)$ across 90 trials in an example PV-Cre mouse injected with AAV-DI0-eYFP. $\boldsymbol{H}$, Spiking activity of the five light-responsive $\mathrm{mPFC}$ PV interneurons (PV-Cre + PV-Cre/NR1f/f mice) in response to application of $3 \mathrm{~s}$ blue light (blue shading; $473 \mathrm{~nm}, 46 \mathrm{~mW} / \mathrm{mm}^{2}$, 3 ms pulses). Light frequencies used: $8,16,24,32,40,48$, and $80 \mathrm{~Hz}$. Only trials with mean FR $>0.1$ spike/s during baseline ( $t=-3$ to $0 \mathrm{~s}$ ) are included. $\boldsymbol{I}, \boldsymbol{J}$, Mean FR of the five light-responsive units in $\boldsymbol{H}$. All five units showed significantly increased spiking in response to blue light application. $\boldsymbol{K}$, Average spike waveform of the five light-responsive units in $\boldsymbol{H}$. Spontaneous (gray) and light-evoked (blue) spike waveforms exhibit very high similarity. $r=$ waveform similarity. ${ }^{*} p<0.05$; ${ }^{* *} p<0.01$; ${ }^{* * *} p<0.001$; two-tailed paired $t$ test. 

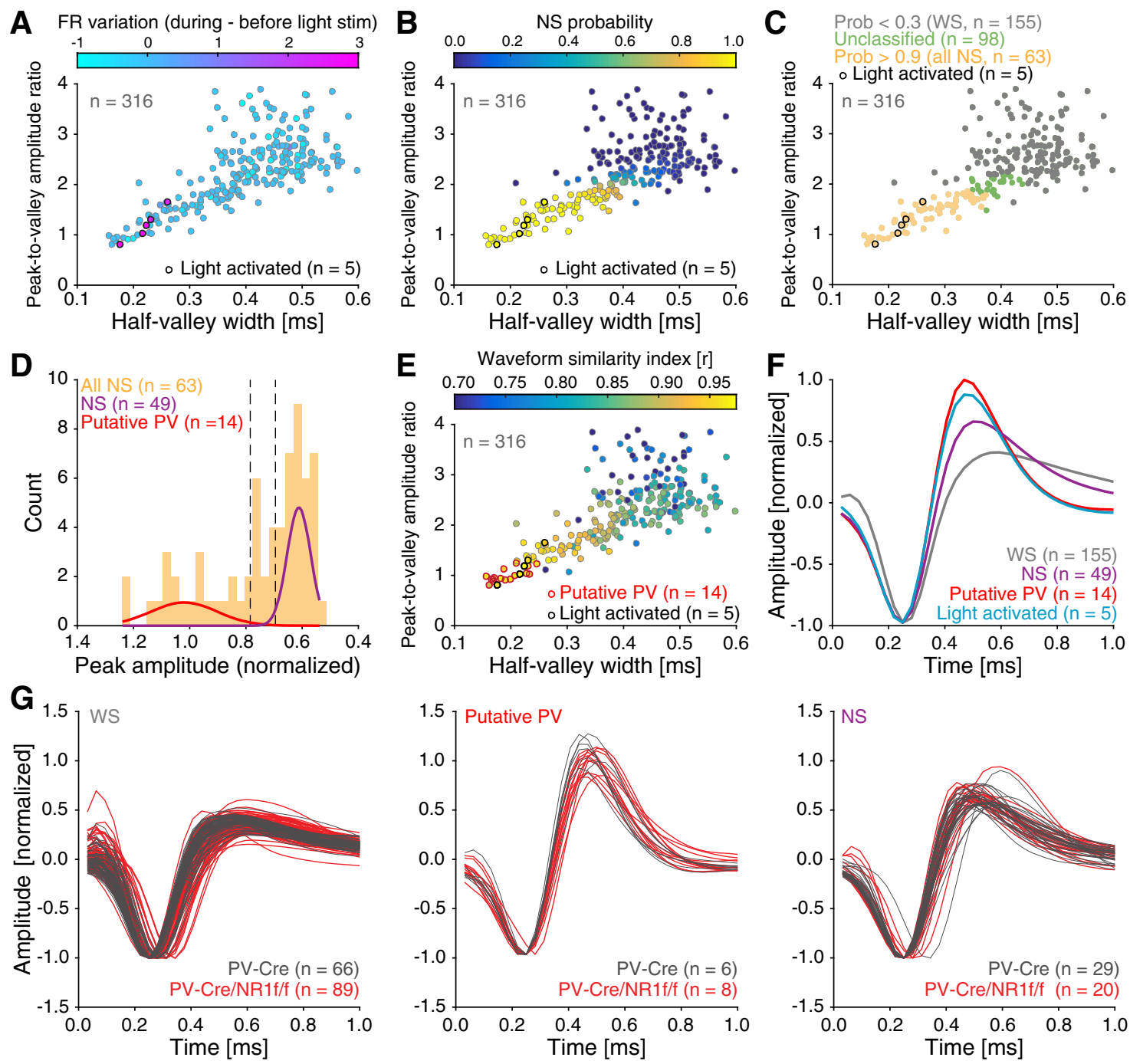

Figure 7. Units classification based on opto-tagging and spike waveform analysis. $A$-G, PV-Cre $(n=4$; gray) and PV-Cre/NR1f/f mice ( $n=3$; red). $A$, Spike waveform features (the mean peak-tovalley amplitude ratio [the ratio between the amplitude of the initial peak and the following trough], and mean half-valley width of the spike waveform) for all individual units $(n=316)$ recorded in mice injected with AAV-DIO-ChR2-mCherry. The firing rate (FR) variation (represented by color bar) elicited by light application was used to identify light-activated units ( $n=5$ units). $\boldsymbol{B}$, For the objective classification of units into WS versus NS, a GMM was fit to the (1) peak-to-valley amplitude ratio and to the (2) half-valley width of the mean spike waveform of the individual units. This identified the NS probability (represented by color bar) for the individual units. C, Classification of WS and NS units after GMM clustering. Units with an NS probability $>0.9$ were classified as NS ("all NS," $n=63)$, and units with an NS probability $<0.3$ were classified as WS $(n=155)$. Units with an intermediate NS probability were unclassified $(n=98$ units) and not included in further analysis. D, A GMM was fit to the peak (normalized amplitude) of the waveform to identify putative PV interneurons within the NS population. NS units with a mean peak amplitude (normalized) $>0.8$ were classified as putative PV interneurons $(n=14)$, and units with mean peak amplitude (normalized) $<0.3$ were classified as NS interneurons $(n=49)$. $\boldsymbol{E}$, Confirmation of the classification of putative PV interneurons by calculation of the spike waveform similarity index (mean correlation between the unit spike waveform and the average spike waveform of the light-activated PV interneurons) for each unit. The putative PV interneurons identified in $\boldsymbol{D}(n=14$; red outline) exhibit very high spike waveform similarity $(r>0.95)$ with light-activated PV interneurons $(n=5$; black outline). $\boldsymbol{F}$, Mean normalized spike waveforms for the classified cell types. WS neurons ( $n=155$ units), putative PV interneurons ( $n=14$ units), NS interneurons ( $n=49$ units), and light-activated PV interneurons ( $n=5$ units). G, Mean normalized spike waveforms for the classified WS (left), putative PV (middle), and NS (right) units.

ketamine-induced state differed from the (altered) baseline spike entrainment in mice lacking NMDAR activity in PV neurons, suggesting disparate circuit underpinnings of the associated high-power broadband $\gamma$ (see further in Discussion).

\section{Discussion}

There is an emerging view that (spontaneous) broadband $\gamma$ oscillations that are measured via a power increase across a wide frequency range at baseline or task-free conditions may be a reflection of increases in the overall level of circuit activity rather than of rhythmic neural activity that is synchronized across neurons (see Sohal and Rubenstein, 2019). While feedback inhibition by cortical PV interneurons is central to rhythmic circuit activity underlying more narrow-band increases in $\gamma$ power, the role in broadband $\gamma$ increases is currently unclear. However, given the important role of PV interneurons in cortical excitation/inhibition balance, reduced PV interneurons function is likely to increase the overall levels of circuit activity, which could cause a broadband power increase across a wide range of frequencies. We here show that mice lacking NMDAR activity in PV neurons display increased power across a (very) broad frequency band, including the broadband $\gamma$ band $(30-80 \mathrm{~Hz})$ and the HFB $(100-$ 
A

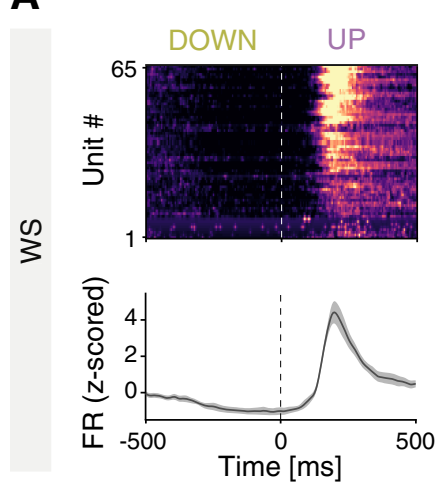

D

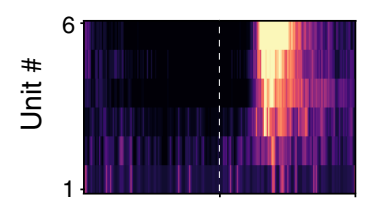

ฉ

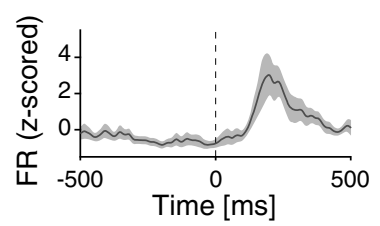

G

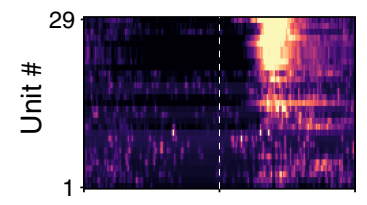

Q

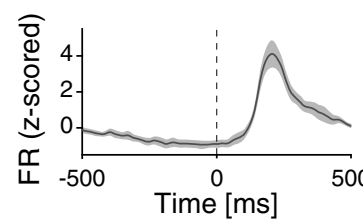

PV-Cre/NR1f/f

DOWN UP
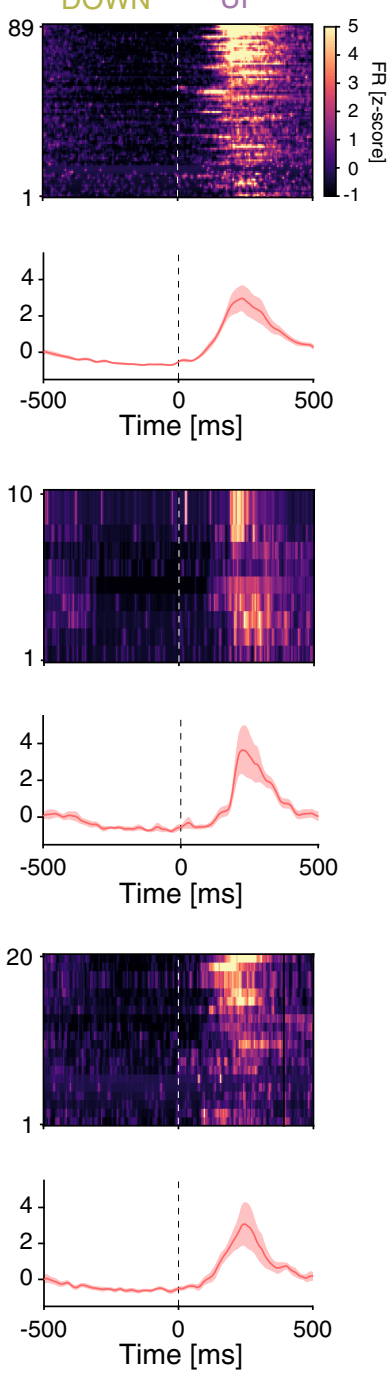

B

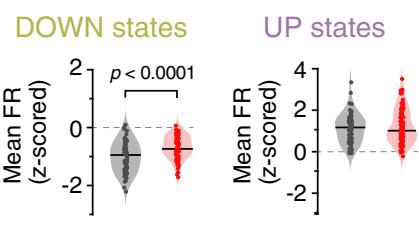

C
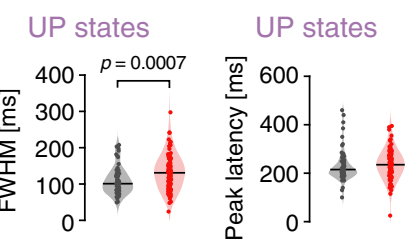

E

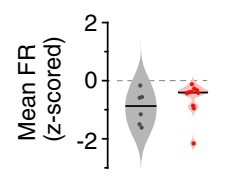

$\mathbf{F}$

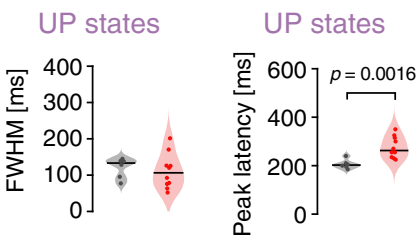

H DOWN states

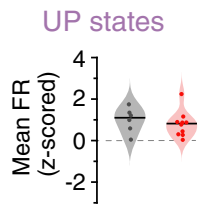

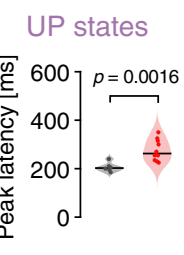

UP states

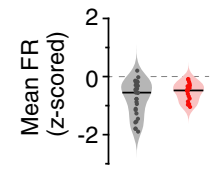

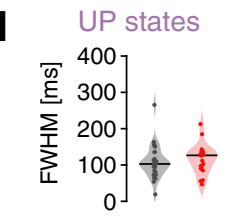
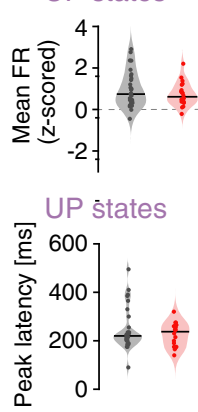

Figure 8. Mice with lack of NMDAR activity in PV neurons display altered temporal dynamics of single-unit activity during DOWN-to-UP state transitions. $A-I, P V-C r e(n=4 ;$ gray) and PVCre/NR1f/f mice $(n=3$; red). A, Firing rate (FR) dynamics of WS neurons during DOWN-to-UP state transition ( -500 to $500 \mathrm{~ms}, 0 \mathrm{~ms}=$ transition [dashed line]). PV-Cre (gray; $n=65$ units) and PV-Cre/NR1f/f mice (red; $n=89$ units). Top, PETH showing mean FR (z-scored) of single WS units. Units are sorted according to their mean FR (z-scored) during the UP state (0-500 ms). Bottom, Mean FR (z-scored) for all WS units. B, C, Firing properties of WS neurons during DOWN-to-UP state transition ( -500 to $500 \mathrm{~ms}$ ). B, The WS FR (z-scored) is significantly higher in PVCre/NR1f/f mice than in PV-Cre mice during DOWN states. DOWN: PV-Cre: -0.95 ; PV-Cre/NR1f/f: $-0.74 ; t=4.575, p<0.0001$; UP: PV-Cre: 1.17; PV-Cre/NR1f/f: 1.01; $t=0.7078, p=0.4802$. C, Left, FWHM of the mean firing curve of individual WS neurons showing significantly increased spike time variability of WS in PV-Cre/NR1f/f mice compared with in PV-Cre mice. Right, Peak latency of the spiking of individual WS neurons. FWHM: PV-Cre: $100.9 \mathrm{~ms}$; PV-Cre/NR1f/f: $130.9 \mathrm{~ms} ; U=1972, p=0.0007$; peak latency: PV-Cre: $215 \mathrm{~ms} ;$ PV-Cre/NR1f/: $235 \mathrm{~ms}$; $U=2391$, $p=0.0661$. D-F, Same as in $\boldsymbol{A}-\boldsymbol{C}$, but for PV interneurons. PV-Cre (gray; $n=6$ units) and PV-Cre/NR1f/f mice (red; $n=10$ units). $\boldsymbol{E}$, The PV FR (z-scored) during DOWN (left) and UP states (right). DOWN: PV-Cre: -0.87 ; PV-Cre/NR1f/f: $-0.41 ; U=18, p=0.2198$; UP: PV-Cre: 1.10; PV-Cre/NR1f/f: $0.81 ; U=20, p=0.3132$. F, Left, FWHM of the mean firing curve of individual PV interneurons. Right, The peak latency of the spiking of individual PV interneurons is significantly increased in PV-Cre/NR1f/f mice compared with that in PV-Cre mice. FWHM: PV-Cre: 133.2 ms; PV-Cre/NR1f/f: 106.3 ms; $U=20, p=0.3132$; peak latency: PV-Cre: $202.5 \mathrm{~ms}$; PV-Cre/NR1f/f: $262.5 \mathrm{~ms} ; U=3, p=0.0016$. G-I, Same as in $A-C$, but for NS putative interneurons. PV-Cre (gray; $n=29$ units) and PV-Cre/NR1f/f mice (red; $n=20$ units). $\boldsymbol{H}$, The NS FR ( $z$-scored) during DOWN (left) and UP states (right). DoWN: PV-Cre: $-0.55 ;$ PV-Cre/NR1f/f: $-0.48 ; t=1.452$, $p=0.1532$; UP: PV-Cre: 0.74 ; PV-Cre/NR1f/f: $0.61 ; t=1.06, p=0.2944$. I, Left, FWHM of the mean firing curve of individual NS interneurons. Right, Peak latency of the spiking of individual NS interneurons. FWHM: PV-Cre: $102.8 \mathrm{~ms}$; PV-Cre/NR1f/f: $126.5 \mathrm{~ms} ; U=244, p=0.3581$; peak latency: PV-Cre: $220 \mathrm{~ms}$; PV-Cre/NR1f/f: $237 \mathrm{~ms} ; \boldsymbol{U}=271, p=0.7052 . \boldsymbol{A}, \boldsymbol{D}, \mathbf{G}, \mathrm{Data}$ are mean \pm

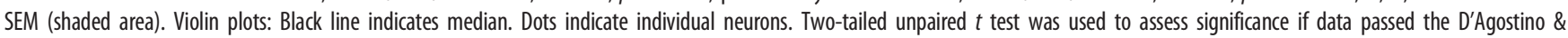
Pearson normality test; if not, the Mann-Whitney test was used. Descriptive statistics for individual mice can be found in Extended Data Figure 8-1.

$150 \mathrm{~Hz}$ ). Importantly, we find the increased high-frequency power to be associated with LFP oscillations and neuronal activity with decreased synchronicity. The main findings distinguishing asynchrony in PV-Cre/NR1f/f mice were as follows: (1) reduced coupling between the $0.5-2 \mathrm{~Hz}$ phase and broadband $\gamma$ amplitude of the LFP; (2) presence of high spectral entropy/high $\mathrm{HF}$ index UP states events with increased $30-150 \mathrm{~Hz}$ power; and (3) a significant and strong positive correlation between the firing rate and the power of both the $\gamma$ band $(30-60 \mathrm{~Hz})$ and the HFB specifically in events with higher HF index. As mentioned earlier, a broad LFP power distribution is considered to reflect a noisy and more irregular LFP caused by disorganized neuronal activity (Uhlhaas et al., 2011), and our single-unit analysis confirmed significantly increased spike latency of PV interneurons and significantly increased variability in the spike-timing of excitatory neurons in mice lacking NMDAR activity in PV neurons 
A

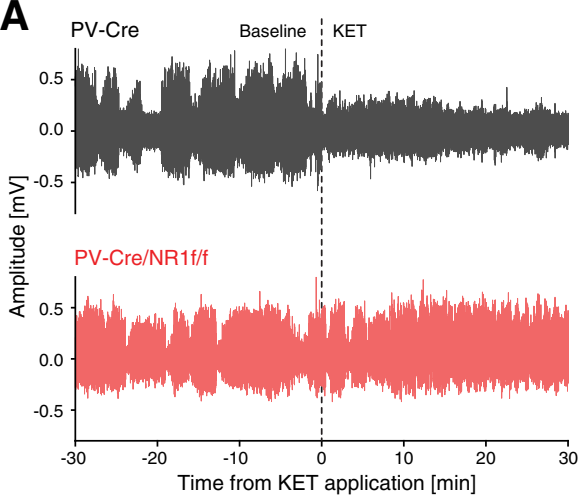

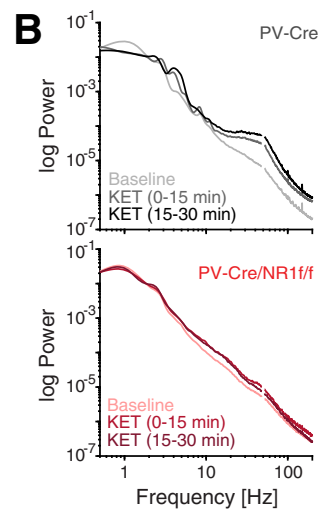

$E_{>}$PV-Cre + KET

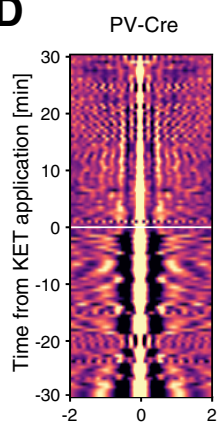

PV-Cre/NR1f/f

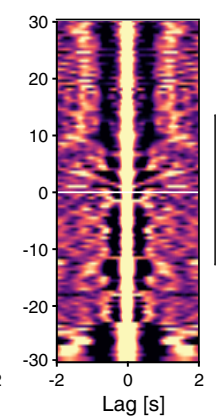

$\sum_{\underline{\underline{E}}} 0.5$

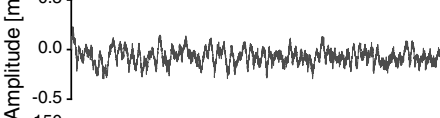

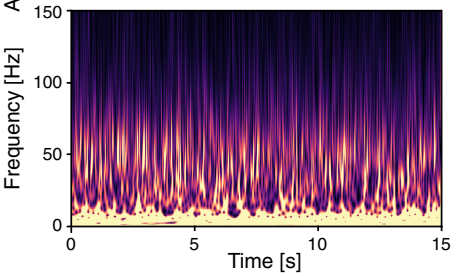

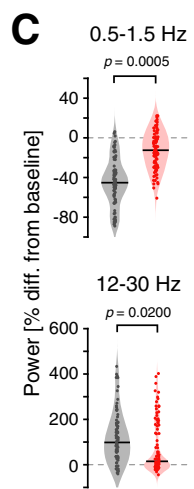

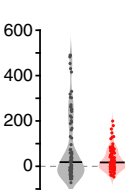

$6-12 \mathrm{~Hz}$
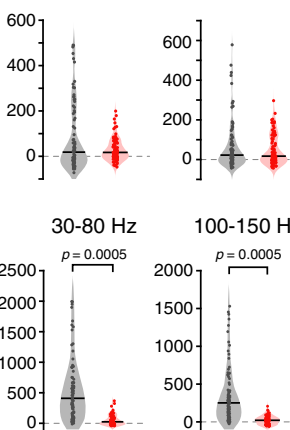

$100-150 \mathrm{~Hz}$

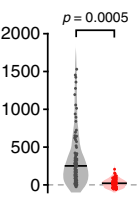

PV-Cre/NR1f/f + KET
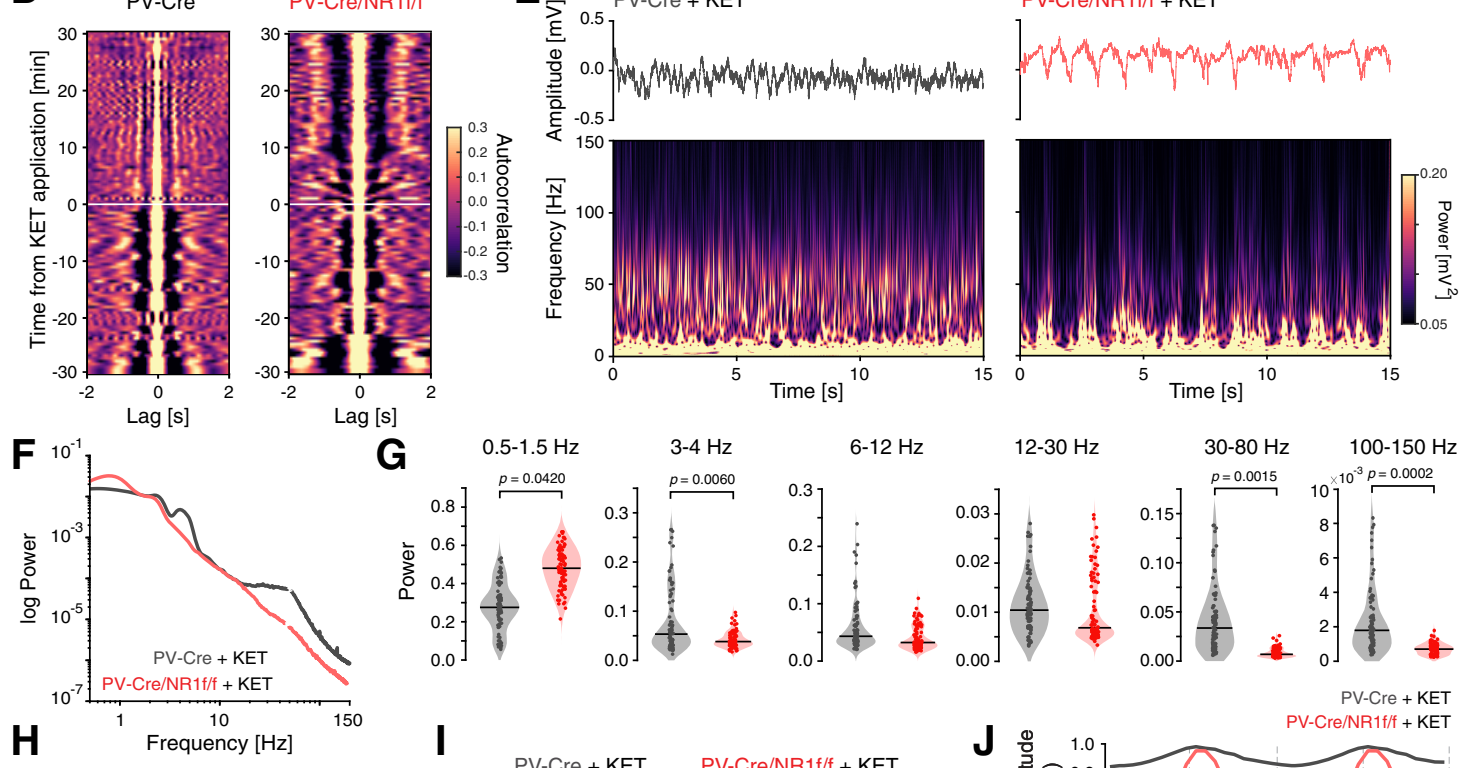

G
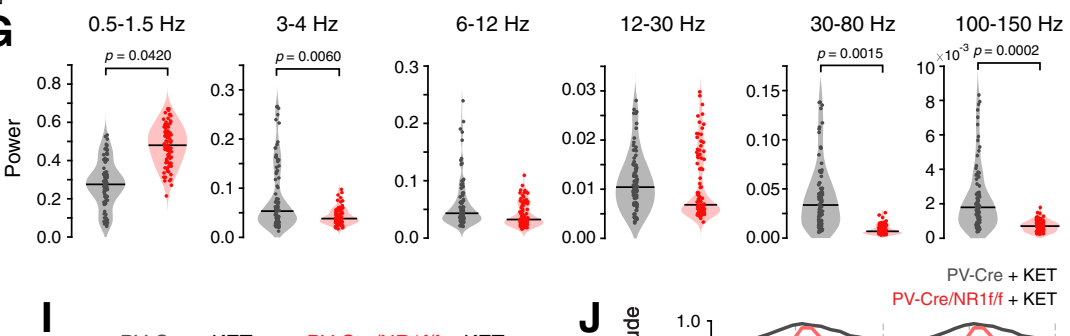

1
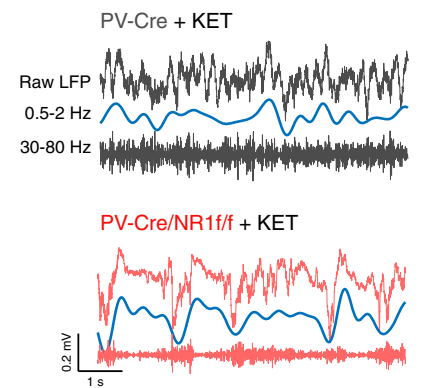
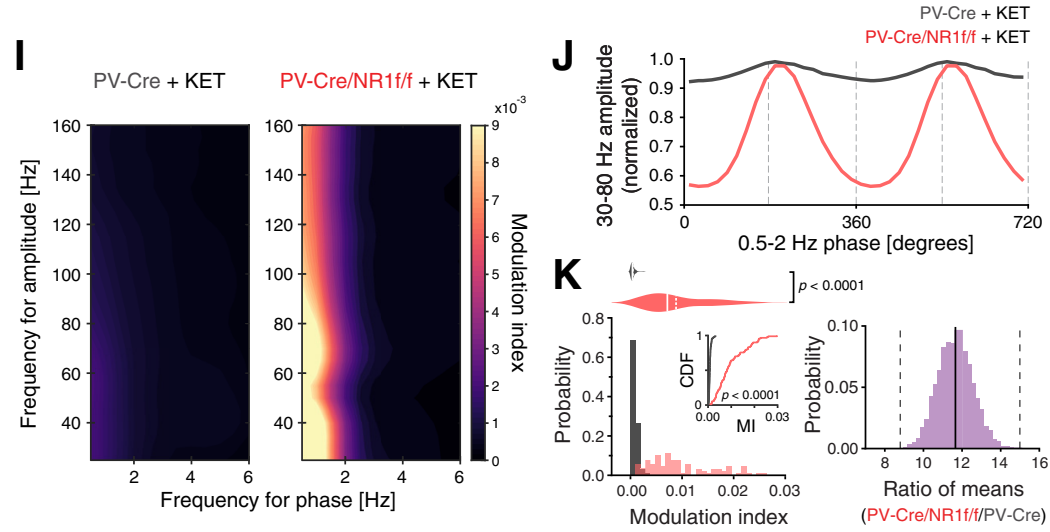

Figure 9. Distinct spectral characteristics of the LFP induced by ketamine. A, Representative unfiltered mPFC LFP traces (60 min) from a PV-Cre (top) and a PV-Cre/NR1f/f (bottom) mouse recorded under urethane anesthesia. Ketamine (KET) was locally applied at $t=0$ (dashed line). In PV-Cre mice, ketamine application switched the LFP oscillations from predominantly low frequencies of high amplitude to higher frequencies of low amplitude. In contrast, ketamine application did not cause any major changes in the mPFC LFP oscillations in PV-Cre/NR1f/f mice. $B$, $C$, $\boldsymbol{F}, \mathbf{G}, \boldsymbol{J}, \boldsymbol{K}, \mathrm{PV}$-Cre ( $n=3$; gray) and PV-Cre/NR1f/f mice ( $n=3$; red). $\boldsymbol{B}$, Mean PSD of the LFP during baseline ( -30 to $0 \mathrm{~min}), 0-15 \mathrm{~min}$, and 15-30 min after ketamine application. $\boldsymbol{C}$, Ketamine induced power change (from baseline) in different frequency bands ( -30 to 0 vs $0-30 \mathrm{~min}$ ). Ketamine application decreased the power in the low- $\delta$ band $(0.5-1.5 \mathrm{~Hz})$ and increased the power in the $\beta(12-30 \mathrm{~Hz})$, broadband $\gamma(30-80 \mathrm{~Hz})$, and HFB $(100-150 \mathrm{~Hz})$ to a significantly larger extent in PV-Cre than PV-Cre/NR1f/f mice. Low- $\delta$ : PV-Cre: $-45.22 \%$; PV-Cre/NR1f/f: $-12.49 \%$; $U=1185, p=0.0005$; high- $\delta$ : PV-Cre: 18.40\%; PV-Cre/NR1f/f: 17.10\%; $U=3980 p=1$; theta: PV-Cre: 22.5\%; PV-Cre/NR1f/f: 17.20\%; $U=4001, p=1 ; \beta$ : PV-Cre: 98.3\%; PV-Cre/NR1f/f: 15\%; $U=3030, p=0.0200$; broadband $\gamma$ : PV-Cre: 408.2\%; PV-Cre/NR1f/f: 24\%; $U=827, p=0.0005$; HFB: PV-Cre: 251.4\%; PV-Cre/NR1f/f: 21.2\%; $U=1054, p=0.0005 . D$, Evolution of the autocorrelograms of the LFP traces in $\boldsymbol{A}$ through time $(60 \mathrm{~min})$, before $(-30$ to $0 \mathrm{~min})$, and after $(0-30 \mathrm{~min})$ ketamine application $(t=0$; white line). Color bar represents the LFP autocorrelation coefficients. Ketamine lowers the LFP autocorrelation in PV-Cre mice, indicating desynchronized LFP oscillations, while the LFP in PV-Cre/NR1f/f mice at large does not change. E-G, Direct comparison of LFP activities after ketamine application between PV-Cre mice and PV-Cre/NR1f/f mice. $\boldsymbol{E}$, Top, $15 \mathrm{~s}$ of the unfiltered LFP traces in $\boldsymbol{A}$ after ketamine application. Bottom, Spectrograms $(0-150 \mathrm{~Hz})$ of the LFP traces, color bar represents LFP power. Ketamine application triggered a state with persistent high-frequency $(>30 \mathrm{~Hz})$ activity of low amplitude in the LFP oscillations in PV-Cre mice, while the low-frequency activity of high amplitude remained in PV-Cre/NR1f/f mice after ketamine application (see also D). $F$, Mean PSD (0-150 Hz) of the LFP after ketamine application (0-30 min), demonstrating decreased power in lower frequencies and increased power in higher frequencies in PV-Cre, but not PV-Cre/NR1f/f mice. G, Power of the different frequency bands after ketamine application (0-30 min). PV-Cre mice display significantly higher power in most frequency bands compared with PV-Cre/NR1f/f mice but significantly lower power in the low- $\delta(0.5-1.5 \mathrm{~Hz})$ band. Low- $\delta$ : PV-Cre: $0.27 \pm 0.06$; PV-Cre/NR1f/f: $0.48 \pm 0.03 ; F_{(1,6)}=15.540, p=0.0462 ;$ high- $\delta$ : PV-Cre: $0.053 \pm 0.032 ;$ PV-Cre/NR1f/f: $0.038 \pm 0.004$; Wald $\chi^{2}=6.43, p=0.0673$; theta: PV-Cre: $0.043 \pm 0.019 ;$ PV-Cre/NR1f/f: $0.032 \pm 0.011$; Wald $\chi^{2}=6.43, p=1 ; \beta$ : PV-Cre: $0.0104 \pm 0.0027 ;$ PV-Cre/NR1f/f: $0.0068 \pm 0.0040 ; F_{(1,6)}=$ 
(Carlén et al., 2012). Earlier works have demonstrated that LFP activity $>80 \mathrm{~Hz}$ can reflect either true neuronal oscillations or spectral leakage of spiking activity (spike "contamination") (Ray and Maunsell, 2011; Scheffer-Teixeira et al., 2013; Rich and Wallis, 2017; Bartoli et al., 2019). Additionally, cortical recordings in nonhuman primates and humans indicate that broadband LFP activity $>80 \mathrm{~Hz}$ can also be observable without the presence of spiking activity and could reflect voltage fluctuations subthreshold to neural firing, notably dependent on NMDAR activity (Leszczyński et al., 2020). We show here that the high spectral entropy/high HF index events in mice lacking NMDAR activity in PV neurons hold spike contamination caused by increased firing rates, leading to increased power in the $30-60 \mathrm{~Hz}$ band and HFB. However, the presence of also low spectral entropy/low HF index events with increased power in the $30-60 \mathrm{~Hz}$ band suggests that oscillations caused by spectral leakage and genuine LFP oscillations coexist within the $\gamma$ band in PV-Cre/ $\mathrm{NR} 1 \mathrm{f} / \mathrm{f}$ mice. It is notable that the asynchronies in PV-Cre/NR1f/ $\mathrm{f}$ mice primarily manifested during the deactivated state, a cortical state marked by synchronous activity (Steriade, 2006; Harris and Thiele, 2011).

PV interneurons have been implicated in the regulation of cortical DOWN and UP state transitions (Zucca et al., 2017), and we show here that the lack of NMDAR activity in PV interneurons is accompanied by increased firing rates of WS neurons during DOWN states, a state typically marked by hyperpolarization. Optogenetic inhibition of cortical PV interneurons has been shown to trigger a DOWN-to-UP transition, which has led to the suggestion that PV firing (although weak) during DOWN states is fundamental to maintain the cortical circuitry in the silent state (Zucca et al., 2017). In line with this, we found the deactivated state to be fragmented in mice lacking NMDAR activity in PV neurons, with significantly reduced UP state duration (plus increased variability of the DOWN state duration in $\mathrm{PV}-\mathrm{Cre} / \mathrm{NR} 1 \mathrm{f} / \mathrm{f}$ mice compared with PV-Cre mice). The reduced hyperpolarization during DOWN states could directly contribute to the decreased power in frequencies $<10 \mathrm{~Hz}$ and increased power in frequencies $>30 \mathrm{~Hz}$, apparent during the deactivated

$\leftarrow$

$0.182, p=1$; broadband $\gamma$ : PV-Cre: $0.0336 \pm 0.0194 ;$ PV-Cre/NR1f/f: $0.0068 \pm 0.0011$; Wald $\chi^{2}=13.354, p=0.0015 ;$ HFB: PV-Cre: $0.0018 \pm 0.0001 ;$ PV-Cre/NR1f/f: $0.0007 \pm$ 0.0003; Wald $\chi^{2}=17.359, p=0.0002$. $\boldsymbol{H}-\boldsymbol{K}$, Phase amplitude comodulation after ketamine application (0-30 min). $\boldsymbol{H}$, Representative unfiltered LFP traces (top), filtered $0.5-2 \mathrm{~Hz}$ (middle), and broadband $\gamma(30-80 \mathrm{~Hz}$; bottom). I, Representative phase-amplitude comodulograms of a PV-Cre (left) and a PV-Cre/NR1f/f mouse (right) after ketamine application (0$30 \mathrm{~min}$ ). Ketamine decreases the modulation of broadband $\gamma$ amplitude by the $0.5-2 \mathrm{~Hz}$ phase in PV-Cre, but not in PV-Cre/NR1f/f mice. Color bar represents the MI. J, Mean broadband $\gamma(30-80 \mathrm{~Hz})$ amplitude at different phases of the $0.5-2 \mathrm{~Hz}$ cycles after ketamine application (0-30 min). $\boldsymbol{K}$, Left, Comparison of the probability distribution of the Ml between broadband $\gamma(30-80 \mathrm{~Hz})$ amplitude and the $0.5-2 \mathrm{~Hz}$ phase after ketamine application $(0-$ $30 \mathrm{~min}$ ) shows that most epochs (30 s) in PV-Cre mice have minimal MI $(p<0.0001$, CDF inset) and the PV-Cre mice have significantly decreased comodulation between broadband $\gamma$ amplitude and $0.5-2 \mathrm{~Hz}$ phase compared with PV-Cre/NR1f/f mice (PV-Cre: $0.0009 \pm$ 0.0001; PV-Cre/NR1f/f: $0.0100 \pm 0.0023$; Wald $\chi^{2}=195.638, p<0.00001$, LMM; right, Cl from bootstrap analysis: $[8.77,15.29])$. C, G, Black lines indicate median. Dots indicate $30 \mathrm{~s}$ epochs. Dashed line indicates baseline. $\boldsymbol{K}$ (violin plots), Solid line indicates mean. Dashed line indicates median. $\boldsymbol{K}$ (bootstrap plot), Solid line indicates mean. Dashed line indicates $95 \% \mathrm{Cl}$. Two-tailed unpaired $t$ test was used to assess significance if data passed the D'Agostino \& Pearson normality test; if not, the Mann-Whitney test was used. The Kolmogorov-Smirnov test was used to assess significance between CDFs, and complemented with an LMM and a bootstrap analysis to assess significance between PV-Cre and PV-Cre/NR1f/f mice while accounting for intragroup variability. Complementary statistical information can be found in Extended Data Figure 9-1. states in mice lacking NMDAR activity in PV neurons. NMDAR hypofunction in PV neurons could give rise to increased excitatory activity through different mechanisms. Reduced postsynaptic NMDAR activity in PV neurons could reduce the PV excitability, and hence the PV activity (Korotkova et al., 2010; Carlén et al., 2012). More recent findings suggest that presynaptic activity of NMDARs in PV interneurons enhances the probability of GABA release, including in the PFC (Pafundo et al., 2018). Reduced presynaptic NMDAR activity would reduce the IPSCs and the strength of PV-to-pyramidal inhibition (Jadi et al., 2016; Pafundo et al., 2018). As we here delete the ubiquitous NR1 subunit, both presynaptic and postsynaptic changes in NMDAR activity could plausibly contribute to the observed alterations.

Local ketamine application resulted in a desynchronized state, characterized by lower amplitude and higher frequencies of LFP oscillations, and reduced modulation of $\gamma$ amplitude by the 0.5 $2 \mathrm{~Hz}$ phase in mice with intact NMDAR activity in PV neurons. Interestingly, systemic ketamine administration has been shown to alter sleep-like oscillations (induced by pentobarbital) in the frontoparietal cortex in similar ways, and the associated LFP changes were found to be related to ketamine-induced alterations in the thalamic reticular nucleus and thalamocortical networks (Mahdavi et al., 2020). Thus, our results on reduced synchronization of LFP activity might be similar to the effect of systemic ketamine of thalamic networks (Mahdavi et al., 2020), given that local application of ketamine affects connected structures (Kulikova et al., 2012). In agreement, local blockade of NMDAR activity by application of MK-801 has been demonstrated to propagate through connected neural circuits (Lee et al., 2017). Previous in vivo studies have demonstrated that NMDAR antagonists, including ketamine, increase the firing of prefrontal neurons (Wood et al., 2012; Molina et al., 2014). We show here that local ketamine application increases the firing of both prefrontal WS neurons and NS interneurons. The observation that the power of cortical high-frequency LFP oscillations $(50-180 \mathrm{~Hz})$ and the firing rates in the local circuits positively correlate with the entrainment of spikes to the LFP in the 50$180 \mathrm{~Hz}$ band suggests that local neuronal activity underlies the generation of high-frequency LFP oscillations (Watson et al., 2018). Analysis of the entrainment of spikes to the LFP can thus offer insight into mechanisms underlying high-frequency LFP oscillations. In the current study, the baseline spiking in mice with intact NMDAR activity in PV neurons was predominantly entrained by LFP oscillations in the $50-180 \mathrm{~Hz}$ band, with local ketamine application shifting the entrainment to higher frequencies $(>200 \mathrm{~Hz})$. However, mPFC neurons in mice lacking NMDAR activity in PV neurons were entrained by LFP oscillations of a broader frequency range (and particularly by lower frequencies), and ketamine did not change the spike entrainment. Both PV-Cre/NR1f/f mice and mice treated with ketamine display increased power of broadband $\gamma$; however, the entrainment of spikes to the LFP differs, which suggests that the enhanced broadband $\gamma$ power observed in the two animal groups arise by disparate mechanisms. Acute (NMDAR antagonists) and longterm (genetic) PV hypofunction, respectively, clearly entail distinct circuit alterations, the latter including not only activity alterations but also structural changes. Dendritic and axonal structural changes in PV interneuron can give rise to lasting disorganization of local network activities (del Pino et al., 2013; Hamm et al., 2017; Guyon et al., 2021), alteration not replicated by ketamine-induced suppression of PV inhibition (Hamm et al., 2017). 
A

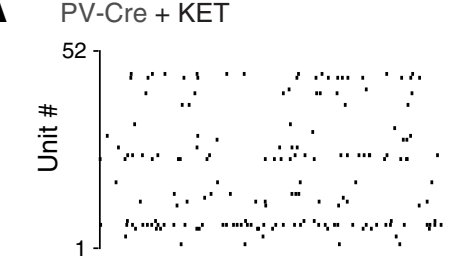

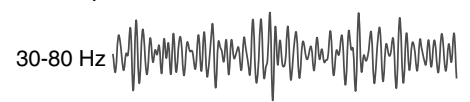
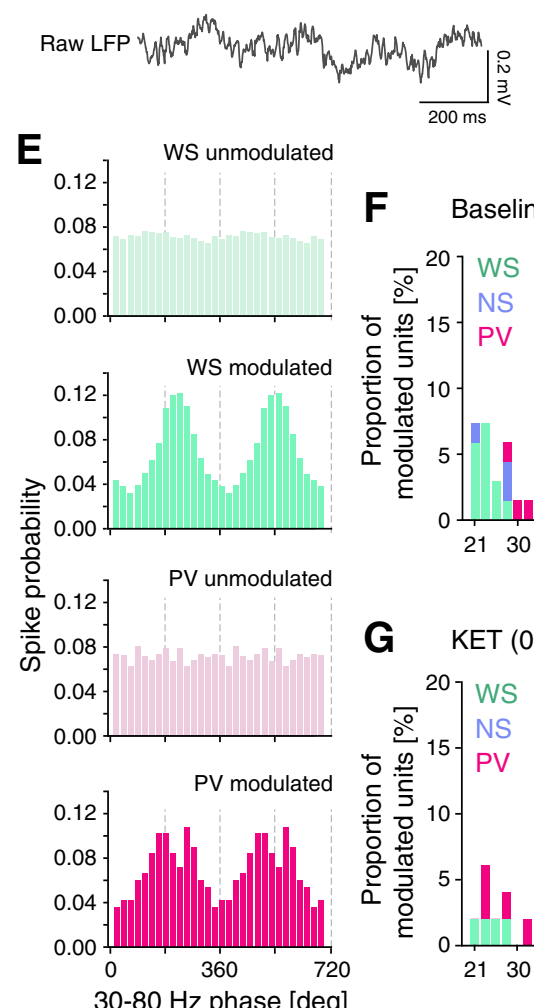

\section{F Baseline (-30-0 min)}

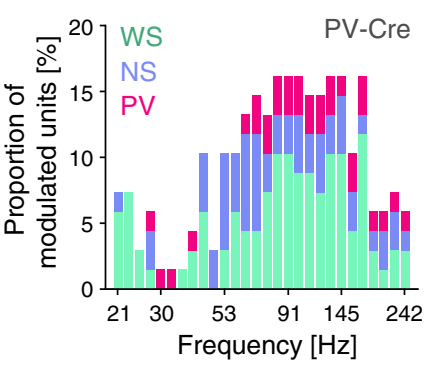

G $\operatorname{KET}(0-30 \mathrm{~min})$

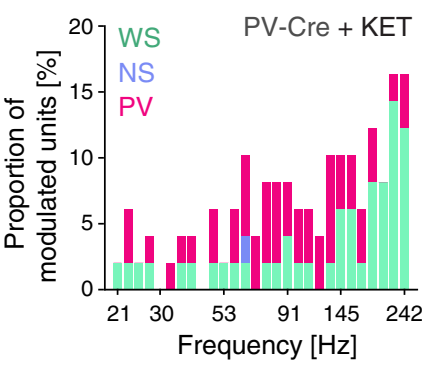

B PV-Cre/NR1f/f + KET

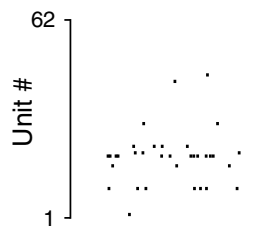

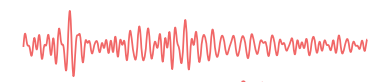

D NS+

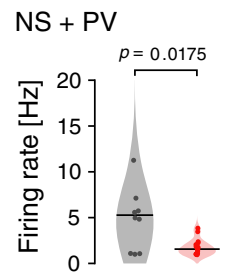

C WS $p<0.0001$

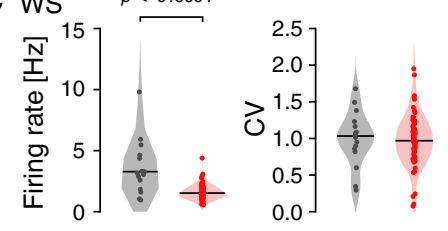

PV-Cre + KET PV-Cre/NR1f/f + KET

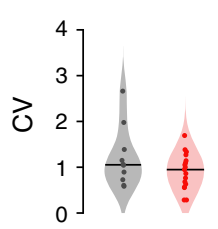

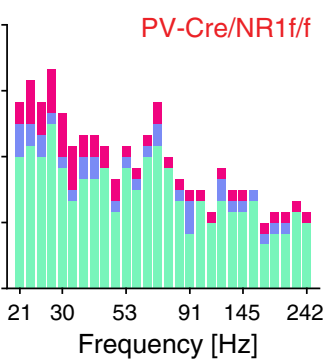

(PV-Cre) - (PV-Cre/NR1f/f)

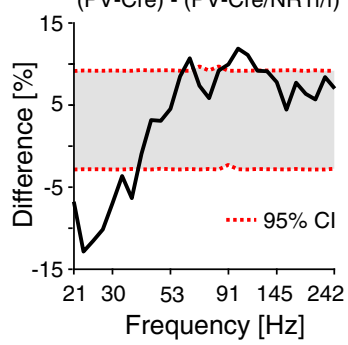

Frequency $[\mathrm{Hz}]$

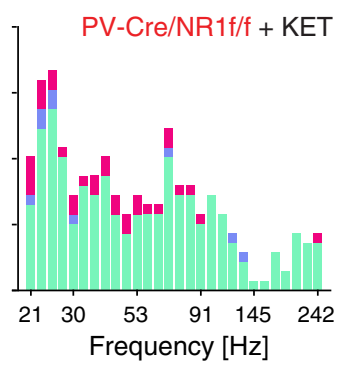

(PV-Cre) - (PV-Cre/NR1f/f)

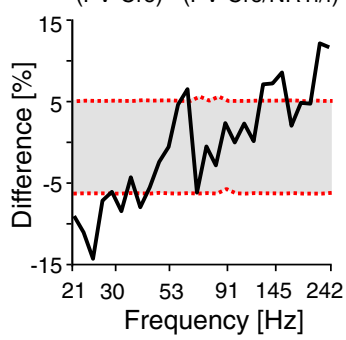

Figure 10. Firing patterns and spike-LFP entrainment during baseline and after ketamine application. $\boldsymbol{A}, \boldsymbol{B}$, Representative $(1 \mathrm{~s})$ single-unit activity (top), filtered LFP in the $30-80 \mathrm{~Hz}$ band (middle), and raw LFP traces (bottom) for a PV-Cre (A) and a PV-Cre/NR1f/f mouse (B) after ketamine application. C, D, F, G, PV-Cre $(n=3$; gray) and PV-Cre/NR1f/f mice $(n=3$; red). $\boldsymbol{C}$, FR properties of WS neurons (PV-Cre: $n=17$ units; PV-Cre/NR1f/f: $n=69$ units) after ketamine application (0-30 min). Left, The FR of WS neurons is significantly higher in PV-Cre mice than in PV-Cre/NR1f/f mice. Right, The CV of the ISI does not differ between PV-Cre and PV-Cre/NR1f/f mice. FR: PV-Cre: 3.28; PV-Cre/NR1f/f: 1.52; U = 189, $p<0.0001 ;$ CV: PV-Cre: 1.03; PV-Cre/ NR1f/f: 0.97; $U=547, p=0.6751$. D, FR properties of NS+PV putative interneurons (PV-Cre: $n=10$ units; PV-Cre/NR1f/f: $n=17$ units) after ketamine application (0-30 min). Left, The FR of $\mathrm{NS}+\mathrm{PV}$ interneurons is significantly higher in PV-Cre mice than in PV-Cre/NR1f/f mice after ketamine application. Right, The CV of the ISI does not differ between PV-Cre and PV-Cre/NR1f/f mice. FR: PV-Cre: 5.27; PV-Cre/NR1f/f: 1.56; $U=38, p=0.0175$; CV: PV-Cre: 1.05; PV-Cre/NR1f/f: 0.95; $U=64 p=0.3093$. E, Histograms of the spike modulation by broadband $\gamma(30-80 \mathrm{~Hz}$ ) phase. Four representative single neurons: light green represents nonmodulated WS; green represents modulated WS; light pink represents nonmodulated PV; pink represents modulated PV. $\boldsymbol{F}, \boldsymbol{G}$, Spike-LFP entrainment in PV-Cre (left) and PV-Cre/NR1f/f (middle) mice during baseline ( -30 to $0 \mathrm{~min})(\boldsymbol{F})$ and after ketamine application $(0-30 \mathrm{~min})(\boldsymbol{G})$. $\boldsymbol{F}$, The proportion of neurons significantly $(p<0.01)$ modulated by different LFP frequencies $(21-242 \mathrm{~Hz}$; log scale). The spiking in PV-Cre mice is predominantly modulated by higher frequencies, and in PV-Cre/NR1f/f mice by lower frequencies. WS (green): PV-Cre: $n=22$ units; PV-Cre/NR1f/f: $n=90$ units; NS (blue): PV-Cre: $n=7$ units; PV-Cre/NR1f/f: $n=12$ units; PV (pink): PV-Cre: $n=3$ units; PV-Cre/ NR1f/f: $n=5$ units. Right, Difference in the proportion of neurons (WS + NS + PV) significantly $(p<0.01)$ modulated by different LFP frequencies (21-242 Hz) between PV-Cre and PV-Cre/ NR1f/f mice. The modulation of spiking by higher LFP frequencies is significantly stronger in PV-Cre than in PV-Cre/NR1f/f mice. G, Ketamine shifts the modulation of spiking in PV-Cre mice to higher LFP frequencies ( $>200 \mathrm{~Hz}$ ) while the spiking in PV-Cre/NR1f/f mice at large is not affected. Right, After ketamine application, the modulation of frequencies $>200 \mathrm{~Hz}$ is significantly stronger in PV-Cre than in PV-Cre/NR1f/f mice. C, D, Black line indicates median. Dots indicate individual neurons. $\boldsymbol{F}, \mathbf{G}$, Bars are stacked. Two-tailed unpaired $t$ test was used to assess significance if data passed the D'Agostino \& Pearson normality test; if not, the Mann-Whitney test was used. Complementary statistical information can be found in Extended Data Figure 10-1.

Sleep and circadian rhythm dysfunctions are comorbidities of schizophrenia. Interestingly, the fragmentation of the deactivated state in the current study parallels findings of fragmentation specifically of NREM sleep in mouse models of schizophrenia involving PV interneuron dysfunction (Phillips et al., 2012). Cortical state transitions under sleep-wake cycles (for review, see Brown and McKenna, 2015) and under urethane anesthesia (Clement et al., 2008) involve complex interactions between cortical and subcortical networks, with notable involvement of cholinergic (Clement et al., 2008; T. Kim et al., 2015; Tikhonova et al., 2018), long-range GABA-ergic PV neurons in the basal forebrain (McKenna et al., 2020; McNally et al., 2020), and GABA-ergic PV neurons in the thalamic reticular nucleus (Steriade, 2006; Cohen et al., 2015; Mahdavi et al., 2020). As an example, optogenetic stimulation of PV neurons in the basal forebrain shortens the NREM sleep states without affecting REM states (McKenna et al., 2020). The global knock-out of NMDAR in PV neurons in PV-Cre/NR1f/f mice could affect long-range 
PV neurons in the basal forebrain and their function in coordination of cortical brain states, possibly contributing to the observed fragmentation of the deactivated states. Clearly, the lack of NMDAR in prefrontal PV interneurons could, in addition, affect the local processing of inputs from subcortical regions regulating arousal and cortical states (Bogart and O'Donnell, 2018; ährlund-Richter et al., 2019). Together, our investigations demonstrate that enhanced broadband $\gamma$ power can be a result of asynchronous neuronal activity and that the power increase can arise by different circuit mechanisms. Our results thus give support for the view that heightened circuit activity resulting from increased excitation/inhibition ratio can be reflected by increased power of broadband $\gamma$ oscillations in the LFP. Importantly, our findings demonstrate that PV interneurons do not only contribute to genuine narrow-band $\gamma$ oscillations that depend on rhythmic feedback inhibition (Cardin et al., 2009; Buzsáki and Wang, 2012), but can also be involved in increased broadband $\gamma$ power associated with cognitive dysfunction.

\section{References}

ährlund-Richter S, Xuan Y, van Lunteren JA, Kim H, Ortiz C, Pollak Dorocic I, Meletis K, Carlén M (2019) A whole-brain atlas of monosynaptic input targeting four different cell types in the medial prefrontal cortex of the mouse. Nat Neurosci 22:657-668.

Bakker R, Tiesinga P, Kötter R (2015) The scalable brain atlas: instant web-based access to public brain atlases and related content. Neuroinformatics 13:353366.

Bartoli E, Bosking W, Chen Y, Li Y, Sheth SA, Beauchamp MS, Yoshor D, Foster BL (2019) Functionally distinct gamma range activity revealed by stimulus tuning in human visual cortex. Curr Biol 29:3345-3358.e7.

Belluscio MA, Mizuseki K, Schmidt R, Kempter R, Buzsáki G (2012) Crossfrequency phase-phase coupling between theta and gamma oscillations in the hippocampus. J Neurosci 32:423-435.

Billingslea EN, Tatard-Leitman VM, Anguiano J, Jutzeler CR, Suh J, Saunders JA, Morita S, Featherstone RE, Ortinski PI, Gandal MJ, Lin R, Liang Y, Gur RE, Carlson GC, Hahn CG, Siegel SJ (2014) Parvalbumin cell ablation of NMDA-R1 causes increased resting network excitability with associated social and self-care deficits. Neuropsychopharmacology 39:1603-1613.

Bogart LJ, O’Donnell P (2018) Multiple long-range inputs evoke NMDA currents in prefrontal cortex fast-spiking interneurons. Neuropsychopharmacology 43:2101-2108.

Börgers C, Kopell N (2005) Effects of noisy drive on rhythms in networks of excitatory and inhibitory neurons. Neural Comput 17:557-608.

Brown RE, McKenna JT (2015) Turning a negative into a positive: ascending GABAergic control of cortical activation and arousal. Front Neurol 6:135.

Buzsáki G, Wang XJ (2012) Mechanisms of gamma oscillations. Annu Rev Neurosci 35:203-225.

Caixeta FV, Cornélio AM, Scheffer-Teixeira R, Ribeiro S, Tort AB (2013) Ketamine alters oscillatory coupling in the hippocampus. Sci Rep 3:2348.

Canolty RT, Knight RT (2010) The functional role of cross-frequency coupling. Trends Cogn Sci 14:506-515.

Cardin JA, Carlén M, Meletis K, Knoblich U, Zhang F, Deisseroth K, Tsai LH, Moore CI (2009) Driving fast-spiking cells induces gamma rhythm and controls sensory responses. Nature 459:663-667.

Cardin JA, Carlén M, Meletis K, Knoblich U, Zhang F, Deisseroth K, Tsai LH, Moore CI (2010) Targeted optogenetic stimulation and recording of neurons in vivo using cell-type-specific expression of Channelrhodopsin2. Nat Protoc 5:247-254.

Carlén M, Meletis K, Siegle JH, Cardin JA, Futai K, Vierling-Claassen D, Rühlmann C, Jones SR, Deisseroth K, Sheng M, Moore CI, Tsai LH (2012) A critical role for NMDA receptors in parvalbumin interneurons for gamma rhythm induction and behavior. Mol Psychiatry 17:537-548.

Cho KK, Hoch R, Lee AT, Patel T, Rubenstein JL, Sohal VS (2015) Gamma rhythms link prefrontal interneuron dysfunction with cognitive inflexibility in Dlx $5 / 6^{+/-}$mice. Neuron 85:1332-1343.

Chouinard S, Poulin J, Stip E, Godbout R (2004) Sleep in untreated patients with schizophrenia: a meta-analysis. Schizophr Bull 30:957-967.
Clement EA, Richard A, Thwaites M, Ailon J, Peters S, Dickson CT (2008) Cyclic and sleep-like spontaneous alternations of brain state under urethane anaesthesia. PLoS One 3:e2004.

Cohen SM, Tsien RW, Goff DC, Halassa MM (2015) The impact of NMDA receptor hypofunction on GABAergic neurons in the pathophysiology of schizophrenia. Schizophr Res 167:98-107.

del Pino I, García-Frigola C, Dehorter N, Brotons-Mas JR, Alvarez-Salvado E, Martínez de Lagrán M, Ciceri G, Gabaldón MV, Moratal D, Dierssen M, Canals S, Marín O, Rico B (2013) Erbb4 deletion from fast-spiking interneurons causes schizophrenia-like phenotypes. Neuron 79:11521168.

Delorme A, Makeig S (2004) EEGLAB: an Open Source toolbox for analysis of single-trial EEG dynamics including independent component analysis. J Neurosci Methods 134:9-21.

Forrest D, Yuzaki M, Soares HD, Ng L, Luk DC, Sheng M, Stewart CL, Morgan JI, Connor JA, Curran T (1994) Targeted disruption of NMDA receptor 1 gene abolishes NMDA response and results in neonatal death. Neuron 13:325-338.

Guyon N, Zacharias LR, van Lunteren JA, Immenschuh J, Fuzik J, Märtin A, Xuan Y, Zilberter M, Kim H, Meletis K, Lopes-Aguiar C, Carlén M (2021) Adult trkB signaling in parvalbumin interneurons is essential to prefrontal network dynamics. J Neurosci. Advance online publication. Retrieved Feb 16, 2021. doi:10.1523/JNEUROSCI.1848-20.2021.

Hakami T, Jones NC, Tolmacheva EA, Gaudias J, Chaumont J, Salzberg M, O'Brien TJ, Pinault D (2009) NMDA receptor hypofunction leads to generalized and persistent aberrant $\gamma$ oscillations independent of hyperlocomotion and the state of consciousness. PLoS One 4:e6755.

Hamm JP, Peterka DS, Gogos JA, Yuste R (2017) Altered cortical ensembles in mouse models of schizophrenia. Neuron 94:153-167.e8.

Harris KD, Thiele A (2011) Cortical state and attention. Nat Rev Neurosci 12:509-523.

Hauer BE, Pagliardini S, Dickson CT (2019) The reuniens nucleus of the thalamus has an essential role in coordinating slow-wave activity between neocortex and hippocampus. eNeuro 6:ENEURO.0365-19.2019.

Homayoun H, Moghaddam B (2007) NMDA receptor hypofunction produces opposite effects on prefrontal cortex interneurons and pyramidal neurons. J Neurosci 27:11496-11500.

Hudson MR, Sokolenko E, O’Brien TJ, Jones NC (2020) NMDA receptors on parvalbumin-positive interneurons and pyramidal neurons both contribute to MK-801 induced gamma oscillatory disturbances: complex relationships with behaviour. Neurobiol Dis 134:104625.

Jadi MP, Behrens MM, Sejnowski TJ (2016) Abnormal gamma oscillations in $\mathrm{N}$-methyl-D-aspartate receptor hypofunction models of schizophrenia. Biol Psychiatry 79:716-726.

Ju P, Cui D (2016) The involvement of N-methyl-d-aspartate receptor (NMDAR) subunit NR1 in the pathophysiology of schizophrenia. Acta Biochim Biophys Sin (Shanghai) 48:209-219.

Kennedy A (2020) Mouse brain silhouette. Zenodo. Available at https:// zenodo.org/record/3925919\#.YD0bmlUzaUk.

Kim H, ährlund-Richter S, Wang X, Deisseroth K, Carlén M (2016) Prefrontal parvalbumin neurons in control of attention. Cell 164:208218.

Kim H, Brünner HS, Carlén M (2020) The DMCdrive: practical 3D-printable micro-drive system for reliable chronic multi-tetrode recording and optogenetic application in freely behaving rodents. Sci Rep 10:11838.

Kim T, Thankachan S, McKenna JT, McNally JM, Yang C, Choi JH, Chen L, Kocsis B, Deisseroth K, Strecker RE, Basheer R, Brown RE, McCarley RW (2015) Cortically projecting basal forebrain parvalbumin neurons regulate cortical gamma band oscillations. Proc Natl Acad Sci USA 112:3535-3540.

Kiss T, Feng J, Hoffmann WE, Shaffer CL, Hajós M (2013) Rhythmic theta and delta activity of cortical and hippocampal neuronal networks in genetically or pharmacologically induced N-methyl-D-aspartate receptor hypofunction under urethane anesthesia. Neuroscience 237:255-267.

Korotkova T, Fuchs EC, Ponomarenko A, von Engelhardt J, Monyer H (2010) NMDA receptor ablation on parvalbumin-positive interneurons impairs hippocampal synchrony, spatial representations, and working memory. Neuron 68:557-569.

Kulikova SP, Tolmacheva EA, Anderson P, Gaudias J, Adams BE, Zheng T, Pinault D (2012) Opposite effects of ketamine and deep brain stimulation on rat thalamocortical information processing. Eur J Neurosci 36:34073419 . 
Lee J, Hudson MR, O’Brien TJ, Nithianantharajah J, Jones NC (2017) Local NMDA receptor hypofunction evokes generalized effects on gamma and high-frequency oscillations and behavior. Neuroscience 358:124-136.

Lein ES, Hawrylycz MJ, Ao N, Ayres M, Bensinger A, Bernard A, Boe AF, Boguski MS, Brockway KS, Byrnes EJ, Chen L, Chen L, Chen TM, Chin MC, Chong J, Crook BE, Czaplinska A, Dang CN, Datta S, Dee NR, et al. (2007) Genome-wide atlas of gene expression in the adult mouse brain. Nature 445:168-176.

Leszczyński M, Barczak A, Kajikawa Y, Ulbert I, Falchier AY, Tal I, Haegens S, Melloni L, Knight RT, Schroeder CE (2020) Dissociation of broadband high-frequency activity and neuronal firing in the neocortex. Sci Adv 6:977-989.

Lopes-Aguiar C, Ruggiero RN, Rossignoli MT, de Miranda Esteves I, Peixoto-Santos JE, Romcy-Pereira RN, Leite JP (2020) Long-term potentiation prevents ketamine-induced aberrant neurophysiological dynamics in the hippocampus-prefrontal cortex pathway in vivo. Sci Rep 10:15.

Mahdavi A, Qin Y, Aubry AS, Cornec D, Kulikova S, Pinault D (2020) A single psychotomimetic dose of ketamine decreases thalamocortical spindles and delta oscillations in the sedated rat. Schizophr Res 222:362-374.

Manning JR, Jacobs J, Fried I, Kahana MJ (2009) Broadband shifts in local field potential power spectra are correlated with single-neuron spiking in humans. J Neurosci 29:13613-13620.

Massi L, Lagler M, Hartwich K, Borhegyi Z, Somogyi P, Klausberger T (2012) Temporal dynamics of parvalbumin-expressing axo-axonic and basket cells in the rat medial prefrontal cortex in vivo. J Neurosci 32:1649616502.

McKenna JT, Zielinski MR, McCarley RW (2017) Neurobiology of REM sleep, NREM sleep homeostasis, and gamma band oscillations. In: Sleep disorders medicine (Chokroverty S, ed), pp 55-77. New York: Springer.

McKenna JT, Thankachan S, Uygun DS, Shukla C, McNally JM, Schiffino FL, Cordeira J, Katsuki F, Zant JC, Gamble MC, Deisseroth K, McCarley RW, Brown RE, Strecker RE, Basheer R (2020) Basal forebrain parvalbumin neurons mediate arousals from sleep induced by hypercarbia or auditory stimuli. Curr Biol 30:2379-2385.e4.

McNally JM, Aguilar DD, Katsuki F, Radzik LK, Schiffino FL, Uygun DS, McKenna JT, Strecker RE, Deisseroth K, Spencer KM, Brown RE (2020) Optogenetic manipulation of an ascending arousal system tunes cortical broadband gamma power and reveals functional deficits relevant to schizophrenia. Mol Psychiatry. Advance online publication. Retrieved Jul 20, 2020. doi: 10.1038/s41380-020-0840-3.

Molina LA, Skelin I, Gruber AJ (2014) Acute NMDA receptor antagonism disrupts synchronization of action potential firing in rat prefrontal cortex. PLoS One 9:e85842.

Pafundo DE, Miyamae T, Lewis DA, Gonzalez-Burgos G (2018) Presynaptic effects of N-methyl-D-aspartate receptors enhance parvalbumin cellmediated inhibition of pyramidal cells in mouse prefrontal cortex. Biol Psychiatry 84:460-470.

Pagliardini S, Gosgnach S, Dickson CT (2013) Spontaneous sleep-like brain state alternations and breathing characteristics in urethane anesthetized mice. PLoS One 8:e70411.

Phillips KG, Bartsch U, McCarthy AP, Edgar DM, Tricklebank MD, Wafford KA, Jones MW (2012) Decoupling of sleep-dependent cortical and hippocampal interactions in a neurodevelopmental model of schizophrenia. Neuron 76:526-533.

Picard N, Takesian AE, Fagiolini M, Hensch TK (2019) NMDA 2A receptors in parvalbumin cells mediate sex-specific rapid ketamine response on cortical activity. Mol Psychiatry 24:828-838.

Pinault D (2008) N-Methyl D-aspartate receptor antagonists ketamine and MK-801 induce wake-related aberrant $\gamma$ oscillations in the rat neocortex. Biol Psychiatry 63:730-735.

Puchades MA, Csucs G, Ledergerber D, Leergaard TB, Bjaalie JG (2019) Spatial registration of serial microscopic brain images to three-dimensional reference atlases with the QuickNII tool. PLoS One 14:e0216796.

Puig MV, Ushimaru M, Kawaguchi Y (2008) Two distinct activity patterns of fast-spiking interneurons during neocortical UP states. Proc Natl Acad Sci USA 105:8428-8433.

Ray S, Maunsell JH (2011) Different origins of gamma rhythm and highgamma activity in macaque visual cortex. PLoS Biol 9:e1000610.
Rich EL, Wallis JD (2017) Spatiotemporal dynamics of information encoding revealed in orbitofrontal high-gamma. Nat Commun 8:1139.

Rivolta D, Heidegger T, Scheller B, Sauer A, Schaum M, Birkner K, Singer W, Wibral M, Uhlhaas PJ (2015) Ketamine dysregulates the amplitude and connectivity of high-frequency oscillations in cortical-subcortical networks in humans: evidence from resting-state magnetoencephalography-recordings. Schizophr Bull 41:1105-1114.

Saleem AB, Chadderton P, Apergis-Schoute J, Harris KD, Schultz SR (2010) Methods for predicting cortical UP and DOWN states from the phase of deep layer local field potentials. J Comput Neurosci 29:49-62.

Scheffer-Teixeira R, Belchior H, Leao RN, Ribeiro S, Tort AB (2013) On high-frequency field oscillations $(>100 \mathrm{~Hz})$ and the spectral leakage of spiking activity. J Neurosci 33:1535-1539.

Schmitzer-Torbert N, Jackson J, Henze D, Harris K, Redish AD (2005) Quantitative measures of cluster quality for use in extracellular recordings. Neuroscience 131:1-11.

Schomburg EW, Fernández-Ruiz A, Mizuseki K, Berényi A, Anastassiou CA, Koch C, Buzsáki G (2014) Theta phase segregation of input-specific gamma patterns in entorhinal-hippocampal networks. Neuron 84:470485.

Sohal VS, Rubenstein JL (2019) Excitation-inhibition balance as a framework for investigating mechanisms in neuropsychiatric disorders. Mol Psychiatry 24:1248-1257.

Steriade M (2006) Grouping of brain rhythms in corticothalamic systems. Neuroscience 137:1087-1106.

Steriade M, Nunez A, Amzica F (1993) A novel slow ( $<1 \mathrm{~Hz}$ ) oscillation of neocortical neurons in vivo: depolarizing and hyperpolarizing components. J Neurosci 13:3252-3265

Tahvildari B, Wölfel M, Duque A, McCormick DA (2012) Selective functional interactions between excitatory and inhibitory cortical neurons and differential contribution to persistent activity of the slow oscillation. J Neurosci 32:12165-12179.

Tikhonova TB, Miyamae T, Gulchina Y, Lewis DA, Gonzalez-Burgos G (2018) Cell type- and layer-specific muscarinic potentiation of excitatory synaptic drive onto parvalbumin neurons in mouse prefrontal cortex. eNeuro 5:ENEURO.0208-18.2018-226.

Tort AB, Komorowski R, Eichenbaum H, Kopell N (2010) Measuring phaseamplitude coupling between neuronal oscillations of different frequencies. J Neurophysiol 104:1195-1210.

Tyler E, Kravitz L (2020) Mouse running. Zenodo. Available at https:// zenodo.org/record/3925949\#.YD0bMVUzaUk.

Uhlhaas PJ, Pipa G, Neuenschwander S, Wibral M, Singer W (2011) A new look at gamma? High- $(>60 \mathrm{~Hz}) \gamma$-band activity in cortical networks: function, mechanisms and impairment. Prog Biophys Mol Biol 105:1428.

Uhlhaas PJ, Singer W (2015) Oscillations and neuronal dynamics in schizophrenia: the search for basic symptoms and translational opportunities. Biol Psychiatry 77:1001-1009.

Valero M, Averkin RG, Fernandez-Lamo I, Aguilar J, Lopez-Pigozzi D, Brotons-Mas JR, Cid E, Tamas G, Menendez de la Prida L (2017) Mechanisms for selective single-cell reactivation during offline sharpwave ripples and their distortion by fast ripples. Neuron 94:1234-1247. e7.

Watson BO, Ding M, Buzsáki G (2018) Temporal coupling of field potentials and action potentials in the neocortex. Eur J Neurosci 48:2482-2497.

Wood J, Kim Y, Moghaddam B (2012) Disruption of prefrontal cortex large scale neuronal activity by different classes of psychotomimetic drugs. J Neurosci 32:3022-3031.

Yizhar O, Fenno LE, Prigge M, Schneider F, Davidson TJ, O'Shea DJ, Sohal VS, Goshen I, Finkelstein J, Paz JT, Stehfest K, Fudim R, Ramakrishnan C, Huguenard JR, Hegemann P, Deisseroth K (2011) Neocortical excitation/inhibition balance in information processing and social dysfunction. Nature 477:171-178.

Zucca S, D'Urso G, Pasquale V, Vecchia D, Pica G, Bovetti S, Moretti C, Varani S, Molano-Mazón M, Chiappalone M, Panzeri S, Fellin T (2017) An inhibitory gate for state transition in cortex. Elife 6:e26177. 